Homology, Homotopy and Applications, vol.18(2), 2016, pp.133-168

\title{
COFIBRANCY OF OPERADIC CONSTRUCTIONS IN POSITIVE SYMMETRIC SPECTRA
}

\author{
LUÍS ALEXANDRE PEREIRA
}

(communicated by Brooke Shipley)

\begin{abstract}
We show that when using the underlying positive model structure on symmetric spectra one obtains cofibrancy conditions for operadic constructions under much milder hypothesis than one would need for general categories. Our main result provides such an analysis for a key operation, the "relative composition product" $\circ_{\mathcal{O}}$ between right and left $\mathcal{O}$-modules over a spectral operad $\mathcal{O}$, and as a consequence we recover (and usually strengthen) previous results establishing the Quillen invariance of model structures on categories of algebras via weak equivalences of operads, compatibility of forgetful functors with cofibrations and Reedy cofibrancy of bar constructions.

Key to the results above are novel cofibrancy results for $n$ fold smash powers of positive cofibrant spectra (and the relative statement for maps). Roughly speaking, we show that such $n$ fold powers satisfy a (new) type of $\Sigma_{n}$-cofibrancy which can be viewed as "lax $\Sigma_{n}$-free/projective cofibrancy" in that it determines a larger class of cofibrations still satisfying key technical properties of "true $\Sigma_{n}$-free/projective cofibrancy".
\end{abstract}

\section{Introduction}

Operads provide a convenient way to codify many types of algebraic structures on a category $\mathcal{C}$, such as monoids, commutative monoids or, when $\mathcal{C}$ has extra structure, Lie algebras, $E_{n}$-algebras, among others. Indeed, any of these types of structures can be identified with the algebras in $\mathcal{C}$ over a specific operad.

When $\mathcal{C}$ is additionally a suitable model category it is then natural to ask whether the category of algebras over a fixed operad $\mathcal{O}$, denoted $\operatorname{Alg}_{\mathcal{O}}(\mathcal{C})$, inherits a model structure from $\mathcal{C}$ and, moreover, just how compatible such a model structure on $\operatorname{Alg}_{\mathcal{O}}(\mathcal{C})$ is with the underlying model structure on $\mathcal{C}$. Technical reasons then make it desirable for $\mathcal{C}$ and $\operatorname{Alg}_{\mathcal{O}}(\mathcal{C})$ to be cofibrantly generated model categories (briefly, this means (trivial) cofibrations can be built via colimits from certain generating ones), and one quickly finds that the biggest obstacle to tackling the questions above is the

Received March 9, 2015, revised September 17, 2015; published on September 7, 2016. 2010 Mathematics Subject Classification: 55P43, 55P48, 55U35.

Key words and phrases: operad, algebra, positive spectra, model category, power cofibrant, homotopy. Article available at http://dx.doi.org/10.4310/HHA.2016.v18.n2.a7

Copyright (C) 2016, International Press. Permission to copy for private use granted. 
fact that general colimits in $\operatorname{Alg}_{\mathcal{O}}(\mathcal{C})$ are not underlying colimits in $\mathcal{C}$, so that proving properties of the (intended) cofibrations in $\operatorname{Alg}_{\mathcal{O}}(\mathcal{C})$ requires a substantial amount of work.

More generally, related problems occur when studying other natural operadic constructions. Indeed, one of the most compact ways of describing operads is as the monoids over a certain monoidal structure $\circ$, the composition product, and many operadic constructions, such as right modules, left modules and algebras (which are special left modules "concentrated in degree 0"), are then derived from $\circ$. However, o is an unusual monoidal structure which behaves quite differently with respect to each of its variables, in particular preserving colimits in the first variable but not in the second, and one then finds that studying operadic constructions in a model category context naturally requires answering the non-obvious question of which cofibrations are actually preserved by $\circ$, and when.

When dealing with a general model category $\mathcal{C}$ answering the questions above seems to require mild to severe cofibrancy conditions on the operad $\mathcal{O}$ itself (cf. [18]). The main goal of this paper is to prove that for the category $\mathrm{Sp}^{\Sigma}$ of symmetric spectra, however, these questions can be answered while making minimal to no cofibrancy assumptions on $\mathcal{O}$, at least provided one uses the positive $S$ model structure as the underlying model structure on $\mathrm{Sp}^{\Sigma}$.

\subsection{Main results}

Positive model structures on spectra were introduced by Mandell, May, Schwede and Shipley in [11] and soon after used by Shipley in [16] to establish the existence of a projective model structure of symmetric ring spectra where cofibrations are compatible with the forgetful functor from symmetric ring spectra to spectra. Since then, many other results have shown the usefulness of positive structures when studying algebras over an operad, such as the existence of projective model structures for algebras over any simplicial operad shown by Elmendorf and Mandell in [1], strengthened to hold for any spectral operad by Harper in [3], and the compatibility between cofibrations and the forgetful functor for more general operads shown by Harper and Hess in [5].

Our main result, Theorem 1.1 below, follows this trend by establishing a quite thorough control of the way $\circ$ (or more generally, its relative version $\circ_{\mathcal{O}}$ for right and left $\mathcal{O}$-modules) interacts with cofibrations. We encourage the reader daunted by the technical nature of the result to first peruse Section 1.2, where consequences of Theorem 1.1 (including stronger versions of the results mentioned in the previous paragraph) are discussed.

Theorem 1.1. Let $\mathcal{O}$ be an operad in $\mathrm{Sp}^{\Sigma}$ and consider the relative composition product

$$
\operatorname{Mod}_{\mathcal{O}}^{r} \times \operatorname{Mod}_{\mathcal{O}}^{l} \stackrel{-\mathrm{OO}^{-}}{\longrightarrow} \text { Sym }
$$

Regard $\operatorname{Mod}_{\mathcal{O}}^{l}$ as equipped with the projective positive $S$ stable model structure and Sym as equipped with the $S$ stable model structure.

Suppose $f_{2}: M \rightarrow \bar{M}$ is a cofibration between cofibrant objects in $\operatorname{Mod}_{\mathcal{O}}^{l}$. Then if the map $f_{1}: N \rightarrow \bar{N}$ in $\operatorname{Mod}_{\mathcal{O}}^{r}$ is an underlying cofibration (resp., monomorphism) 
in Sym, so is their pushout product with respect to $\circ_{\mathcal{O}}$,

$$
M \circ_{\mathcal{O}} \bar{N} \bigvee_{M \circ \circ^{\circ} N} \bar{M} \circ_{\mathcal{O}} N \stackrel{f_{1} \square^{\circ} \mathcal{O} f_{2}}{\longrightarrow} \bar{M} \circ_{\mathcal{O}} \bar{N}
$$

Further, $f_{1} \square^{\circ} \mathrm{O} f_{2}$ is also a weak equivalence if either $f_{1}$ or $f_{2}$ is.

Technically speaking, most of the ingredients needed for our proof of Theorem 1.1 are adapted from arguments used in $[\mathbf{1}, \mathbf{3}, \mathbf{5}]$ to prove the original versions of the results which we recover in Section 1.2. However, two important new ingredients deserve special mention.

The first of these is found in Proposition 5.20, which extends crucial filtrations of certain pushouts in $\operatorname{Alg}_{\mathcal{O}}(\mathcal{C})$ used in $[\mathbf{1}, \mathbf{3}, \mathbf{5}]$ by still providing such filtrations after composing with $M \circ_{\mathcal{O}}(-)$ for some $M \in \operatorname{Mod}_{\mathcal{O}}^{r}$. Note that as we do not assume $\mathcal{C}=\mathrm{Sp}^{\Sigma}$, these filtrations should be relevant in a general setting.

The second ingredient is a more thorough characterization of what makes positive model structures so convenient. It is well known that, for $A$ any $\Sigma_{n}$-spectrum and $X$ a positive $S$ cofibrant spectrum, there is a canonical weak equivalence

$$
\left(A \wedge X^{\wedge n}\right)_{\Sigma_{n}} \sim\left(A \wedge X^{\wedge n}\right)_{h \Sigma_{n}}
$$

and, indeed, this key result essentially suffices to carry out the proofs in $[\mathbf{1 6}, \mathbf{1}]$. However, since (1) makes no explicit reference to cofibrations, one quickly finds it insufficient when trying to establish cofibrancy results in $\mathrm{Alg}_{\mathcal{O}}$. The natural way to fix this would be to guess that (1) ought to be a consequence of $X^{\wedge n}$ being built from free $\Sigma_{n}$-cells, or put in model category terminology, it being (genuinely) $\Sigma_{n}$-cofibrant (indeed, were that the case combining Remark 2.23 with [9, Thm 5.3.7] would yield (1)). Unfortunately, this turns out to be false (cf. [4]; also, check Remark 2.19), though fortunately not by much. ${ }^{1}$ Indeed, our second ingredient is a (new) type of "lax $\Sigma_{n^{-}}$ cofibrancy" in $\left(\mathrm{Sp}^{\Sigma}\right)^{\Sigma_{n}}$, which we formally call $S \Sigma$-inj $\Sigma_{n}$-proj cofibrancy, such that (i) $X^{\wedge n}$ is "lax $\Sigma_{n}$-cofibrant" for positive cofibrant $X$; (ii) "lax $\Sigma_{n}$-cofibrations" share the key technical properties of (genuine) $\Sigma_{n}$-cofibrations. The formal results follow.

Theorem 1.2. Let $\mathrm{Sp}^{\Sigma}$ be equipped with the positive $S$ stable model structure and $\left(\mathrm{Sp}^{\Sigma}\right)^{\Sigma_{n}}$ with the $S \Sigma$-inj $\Sigma_{n}$-proj stable model structure.

Then for $f: A \rightarrow B$ a cofibration in $\mathrm{Sp}^{\Sigma}$ its $n$-fold pushout product

$$
f^{\square n}: Q_{n-1}^{n}(f) \rightarrow B^{\wedge n}
$$

is a cofibration in $\left(\mathrm{Sp}^{\Sigma}\right)^{\Sigma_{n}}$, which is a weak equivalence if $f$ is.

Furthermore, if $A$ is cofibrant in $\mathrm{Sp}^{\Sigma}$ then $Q_{n-1}^{n}(f)$ (resp., $f^{\wedge n}: A^{\wedge n} \rightarrow B^{\wedge n}$ ) is cofibrant (resp., cofibration between cofibrant objects) in $\left(\mathrm{Sp}^{\Sigma}\right)^{\Sigma_{n}}$.

Theorem 1.3. Consider the bifunctor

$$
\left(\mathrm{Sp}^{\Sigma}\right)^{G} \times\left(\mathrm{Sp}^{\Sigma}\right)^{G} \stackrel{-\wedge_{G}-}{\longrightarrow} \mathrm{Sp}^{\Sigma}
$$

where the first copy of $\left(\mathrm{Sp}^{\Sigma}\right)^{G}$ is regarded as equipped with the $S \Sigma$-inj G-proj stable model structure. Then $\wedge_{G}$ is a left Quillen bifunctor if either:

\footnotetext{
${ }^{1}$ In fact, such a result was "proven" in the author's thesis via an induction argument using incorrect base cofibrancy claims. A key impetus for this paper is to correct those base claims.
} 
(a) Both the second $\left(\mathrm{Sp}^{\Sigma}\right)^{G}$ and the target $\mathrm{Sp}^{\Sigma}$ are equipped with the respective monomorphism stable model structures;

(b) Both the second $\left(\mathrm{Sp}^{\Sigma}\right)^{G}$ and the target $\mathrm{Sp}^{\Sigma}$ are equipped with the respective $S$ stable model structures.

In terms of the existent literature, Theorems 1.2 and 1.3(a) are most closely related to [4, Props. $4.28^{*}, 4.29^{*}$, which they both significantly generalize and improve on from a technical standpoint (cf. Remark 2.24). Further, Theorem 1.3 is strongly motivated by $\left[\mathbf{9}\right.$, Thm 5.3.7] (which, as hinted at above, implies the (genuine) $\Sigma_{n}$-cofibration analogue result).

\subsection{Consequences}

In this section we list a series of less technical results that can easily be deduced from Theorem 1.1 (or, in the case of the first part of Theorem 1.4, its proof).

Theorem 1.4. Let $\mathcal{O}$ be any operad in $\mathrm{Sp}^{\Sigma}$, and let $\mathrm{Sp}^{\Sigma}$, Sym be equipped with the respective positive $S$ stable model structures.

Then the respective projective positive $S$ model structures on $\mathrm{Alg}_{\mathcal{O}}$, $\operatorname{Mod}_{\mathcal{O}}^{l}$ exist and are simplicial model structures.

Further, if $\mathcal{O} \rightarrow \overline{\mathcal{O}}$ is a stable equivalence in each degree then the induce-forget adjunctions

$$
\overline{\mathcal{O}} \circ_{\mathcal{O}}(-): \operatorname{Alg}_{\mathcal{O}} \rightleftarrows \operatorname{Alg}_{\overline{\mathcal{O}}}: \mathrm{fgt}, \quad \overline{\mathcal{O}} \circ_{\mathcal{O}}(-): \operatorname{Mod}_{\mathcal{O}}^{l} \rightleftarrows \operatorname{Mod}_{\overline{\mathcal{O}}}^{l}: \text { fgt }
$$

are Quillen equivalences.

In the case of algebras over the commutative operad, Theorem 1.4 was first proven in $[\mathbf{1 6}]$, and for general simplicial operads in $[\mathbf{1}]$. A result nearly identical to Theorem 1.4 was the main result of [3]. Our result is a slight generalization of the latter in the sense that our model structure on $\operatorname{Mod}_{\mathcal{O}}^{l}$ has a larger class of cofibrations (cf. the discussion preceding [3, Thm. 1.3]).

Theorem 1.5. Let $\mathcal{O}$ be an operad in $\mathrm{Sp}^{\Sigma}$ which is $S$ cofibrant in Sym. Then, equipping $\mathrm{Alg}_{\mathcal{O}}, \operatorname{Mod}_{\mathcal{O}}^{l}$ with their respective projective positive $S$ stable model structures and $\mathrm{Sp}^{\Sigma}$, Sym with their respective $S$ stable model structures, the forgetful functors

$$
\text { fgt: } \operatorname{Alg}_{\mathcal{O}} \rightarrow \mathrm{Sp}^{\Sigma}, \quad \text { fgt: } \operatorname{Mod}_{\mathcal{O}}^{l} \rightarrow \operatorname{Sym}
$$

send cofibrations between cofibrant objects to cofibrations between cofibrant objects.

In the case of algebras over the commutative operad Theorem 1.5 was first proven in [16], and extended to algebras and modules over general operads satisfying some cofibrancy conditions in [5]. Our result improves on the latter by relaxing the cofibrancy conditions on the operad.

Theorem 1.6. Suppose Sym is equipped with the positive $S$ stable model structure and consider an operad $\mathcal{O}$ in $\mathrm{Sp}^{\Sigma}$, right $\mathcal{O}$-module $M$ and a left $\mathcal{O}$-module $N$ such that the unit map $\mathcal{I} \rightarrow \mathcal{O}$ (resp., $M$ and $N$ ) is an underlying cofibration (resp., are cofibrant objects) in Sym. Then the bar construction

$$
B_{n}(M, \mathcal{O}, N)=M \circ \mathcal{O}^{\circ n} \circ N
$$

is Reedy cofibrant with respect to the model structure on Sym. 
A very similar result to Theorem 1.6 was first proven in [5]. Our result improves it by using more general cofibrancy conditions and allowing $\mathcal{O}(0) \neq *$.

Remark 1.7. One advantage of Theorems 1.4, 1.5 and 1.6 versus the original results in $[\mathbf{3}, \mathbf{5}]$ that they generalize is that the cofibrancy conditions used are more consistent across results. This makes it easier to use the results in tandem, a relevant feature in upcoming joint work between the author and Kuhn.

Theorem 1.8. Suppose $A$ is projective positive $S$ cofibrant in $\mathrm{Alg}_{\mathcal{O}}$ or, more generally, in $\operatorname{Mod}_{\overline{\mathcal{O}}}^{l}$. Then the functor

$$
\operatorname{Mod}_{\mathcal{O}}^{r} \stackrel{(-) \circ_{\mathcal{O} A}}{\longrightarrow} \mathrm{Sp}^{\Sigma} \quad \text { or, more generally, } \quad \operatorname{Mod}_{\mathcal{O}}^{r} \stackrel{(-) \circ_{\mathcal{O}} A}{\longrightarrow} \text { Sym }
$$

preserves homotopy fiber sequences.

\subsection{Directions for future work}

A key motivation for the work in this paper comes from upcoming joint work between the author and Kuhn where we study certain filtrations built using $M \circ_{\mathcal{O}}(-)$ type functors. Since we need to iterate such functors while obtaining homotopically meaningful constructions (cf. Theorem 1.8), it becomes necessary to understand how those functors interact with cofibrancy conditions.

Additionally, there are two natural directions in which to try to generalize the results in this paper.

The first direction would be to extend Theorem 1.1 to multicategories/colored operads in $\mathrm{Sp}^{\Sigma}$, following [1]. In light of a recent preprint [18] by White and Yau investigating when analogues of Theorems 1.4, 1.5 hold for general categories, this seems likely to be a formal question.

A second direction would be to extend our main results to other categories. A natural candidate for such a generalization is given by the (simplicial) genuine $G$ symmetric spectra of Hausmann [6], as those share the underlying categories used in this paper. Such a generalization is the subject of upcoming joint work between the author and Hausmann.

\subsection{Outline of the paper}

Section 2 introduces the required basic notation and terminology.

Section 3 defines and proves the existence (Theorems 3.6, 3.7, 3.8) of the three model structures on $\left(\mathrm{Sp}^{\Sigma}\right)^{G}$ necessary to formulate Theorems 1.2 and 1.3.

Section 4 proves the key properties of the $S \Sigma$-inj $G$-proj stable model structures featured in Theorems 1.2 and 1.3, namely those results themselves as well as two minor but essential "change of group" results (Propositions 4.1 and 4.2).

Section 5 deals with proving Theorem 1.1 and its "corollaries" Theorems 1.4, 1.5, 1.6 and 1.8. Key to this is Subsection 5.2 and in particular Proposition 5.20, which improves crucial filtration results used in $[\mathbf{1}, \mathbf{3}, \mathbf{5}]$, among others.

Much of the paper, namely Sections 3 and 4, is devoted to building the notion of "lax $\Sigma_{n}$-cofibrancy" needed to state Theorems 1.2 and 1.3 and then proving those results. However, the reader interested only in Theorem 1.1 (or its consequences) should be able to skip ahead to Section 5, provided he is willing to accept Theorems 1.2 and 1.3 (and Propositions 4.1 and 4.2) as given. 


\section{Acknowledgments}

The author would like to thank Nick Kuhn for his encouragement and advice as well as John Harper, Mark Behrens and David White for many useful conversations.

\section{Basic definitions and notation}

We assume the reader is familiar with the basics on symmetric spectra (as found in [9] or [14]) and cover in Sections 2.1, 2.2 and 2.3 only the minimum needed to establish notation and some less standard basic results.

Likewise, we assume the reader is familiar with the basics on cofibrantly generated model categories (as in [8]) and recall in Sections 2.4 and 2.5 only two notions that play a key role for us: injective/projective model structures and left Quillen bifunctors.

\subsection{Pointed $G$-simplicial sets}

Throughout we let $\left(\mathrm{S}_{*}, \wedge, S^{0}\right)$ denote the closed monoidal category of pointed simplicial sets together with its monoidal structure $\wedge$ and unit $S^{0}$.

We will make use of the following standard notation:

- for $A$ a set, $A \cdot(-)$ denotes the (constant) coproduct over $A$ (cf. [10]);

- $\Delta^{k}, \partial \Delta^{k}$ and $\Lambda_{l}^{k}$ denote the standard, boundary and horn (unpointed) simplicial sets (cf. [2, I.1]);

- $X_{+}$denotes the pointed simplicial set obtained by adding a disjoint base point to the (unpointed) simplicial set $X$;

- $S^{n}=\left(\Delta^{1} / \partial \Delta^{1}\right)^{\wedge n}$ denotes the pointed $n$-sphere.

Definition 2.1. Let $G$ be a finite group. The category $\mathrm{S}_{*}^{G}$ of pointed $G$-simplicial sets is the category of functors $G \rightarrow \mathrm{S}_{*}$.

Given $X, Y$ in $\mathrm{S}_{*}^{G}, X \wedge Y$ has a diagonal $G$-action and, giving $S^{0}$ the trivial action, $\wedge$ becomes a monoidal structure in $S_{*}^{G}$. In fact, one has the following.

Proposition 2.2. $\left(\mathrm{S}_{*}^{G}, \wedge, S^{0}\right)$ form a closed symmetric monoidal category.

Further, both the left and right adjoint in the trivial-fixed point adjunction

$$
\text { triv: } \mathrm{S}_{*} \rightleftarrows \mathrm{S}_{*}^{G}:(-)^{G}
$$

are monoidal functors.

The less obvious half of Proposition 2.2 follows since $X \wedge Y=\operatorname{colim}(* \leftarrow X \vee Y \rightarrow$ $X \times Y)$ together with the following (which we will need later).

Proposition 2.3. Any pushout diagram in $\mathrm{S}_{*}^{G}$

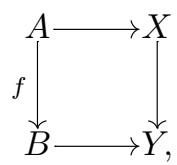

with $f$ a monomorphism remains a pushout diagram after applying $(-)^{G}$.

Proof. Monomorphisms are transfinite compositions of maps adding a single orbit, so that one reduces to $f=G / H \cdot\left(\partial \Delta_{+}^{k} \rightarrow \Delta_{+}^{k}\right)$. The claim is now clear. 
Remark 2.4. By the theory of enriched categories (see, for example, [12, Chap. 3]), Proposition 2.2 implies that $S_{*}^{G}$ is a tensored and cotensored $S_{*}$-enriched category, and hence also simplicially enriched, tensored and cotensored. Explicitly, we note that the mapping space for $X, Y \in \mathrm{S}_{*}^{G}$ is $\operatorname{Map}(X, Y)^{G}$, the $G$-fixed points of the conjugation action on the mapping space of the underlying $X, Y \in \mathrm{S}_{*}$.

\section{2. $\quad G$-spectra}

Throughout $\Sigma$ will denote the usual skeleton of the category of finite sets and bijections. Explicitly, the objects of $\Sigma$ are the sets $\underline{m}=\{1,2, \cdots, m\}$ for $m \geqslant 0$.

Definition 2.5. The category of symmetric sequences in pointed simplicial sets is the category $S_{*}^{\Sigma}$ of functors from $\Sigma$ to $S_{*}$.

Remark 2.6. Unpacking Definition 2.5, a symmetric sequence $X$ consists of a sequence $X_{m}, m \geqslant 0$ of pointed simplicial sets, each with a left $\Sigma_{m}$-action. One then has inclusions $S_{*}^{\Sigma_{m}} \hookrightarrow S_{*}^{\Sigma}$, which we often omit to simplify notation.

Definition 2.7. The tensor product $X \otimes Y$ of $X, Y \in \mathrm{S}_{*}^{\Sigma}$ is defined by

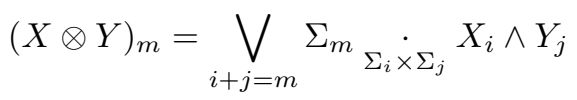

together with the obvious $\Sigma_{m}$-actions.

The following is proven in [9, Sec. 2.2].

Proposition 2.8. $\left(S_{*}^{\Sigma}, \otimes, \mathbb{1}\right)$ form a symmetric monoidal category where the unit $\mathbb{1}$ is the sequence $\mathbb{1}$ such that $\mathbb{1}_{0}=S^{0}$ and $\mathbb{1}_{m}=*$ for $m>0$.

It is well known that the symmetric sequence $S$, the sphere spectrum, defined by $S_{m}=S^{m}$ is a symmetric monoid with respect to $\otimes$. General theory then implies that modules over $S$ themselves form a symmetric monoidal category.

Definition 2.9. The category $\mathrm{Sp}^{\Sigma}$ of symmetric spectra is the category of modules over $S$ in $\mathrm{S}_{*}^{\Sigma}$. The smash product $X \wedge Y$ of $X, Y \in \mathrm{Sp}^{\Sigma}$ is the coequalizer

$$
X \otimes S \otimes Y \rightrightarrows X \otimes Y \rightarrow X \wedge Y .
$$

Remark 2.10. Throughout we will need to consider spectra $X$ such that each level $X_{m}$ is acted on by multiple symmetric groups (e.g., when $X=Y^{\wedge n}$ ). To avoid confusion, we will reserve the letter $m$ for the structure index of spectra.

Definition 2.11. Let $G$ be a finite group. The category $\left(\mathrm{Sp}^{\Sigma}\right)^{G}$ of $G$-spectra is the category of functors $G \rightarrow \mathrm{Sp}^{\Sigma}$.

Just as for pointed simplicial sets, the smash product $X \wedge Y$ of $X, Y \in\left(\mathrm{Sp}^{\Sigma}\right)^{G}$ has a diagonal $G$-action. The following is immediate.

Proposition 2.12. Both $\left(\mathrm{Sp}^{\Sigma}, \wedge, S\right)$ and $\left(\left(\mathrm{Sp}^{\Sigma}\right)^{G}, \wedge, S\right)$ form closed symmetric monoidal categories. Further, all functors in the following adjunctions are monoidal

$$
\begin{aligned}
& S \otimes(-): \mathrm{S}_{*} \rightleftarrows \mathrm{Sp}^{\Sigma}:(-)_{0}, \quad S \otimes(-): \mathrm{S}_{*}^{G} \rightleftarrows\left(\mathrm{Sp}^{\Sigma}\right)^{G}:(-)_{0}, \\
& \text { triv: } \mathrm{Sp}^{\Sigma} \rightleftarrows\left(\mathrm{Sp}^{\Sigma}\right)^{G}:(-)^{G} .
\end{aligned}
$$


Remark 2.13. The theory of enriched categories [12, Chap. 3] implies that $\left(\mathrm{Sp}^{\Sigma}\right)^{G}$ is enriched, tensored and cotensored over both $\mathrm{S}_{*}$ and $\mathrm{Sp}^{\Sigma}$, and hence also simplicially enriched, tensored and cotensored.

\section{3. $\quad S$ stable and positive $S$ stable model structures on $\mathrm{Sp}^{\Sigma}$}

Definition 2.14. The $S$ stable model structure (resp., positive $S$ stable model structure) on $\mathrm{Sp}^{\Sigma}$ is the cofibrantly generated model structure such that

- the generating cofibrations are the maps

$$
S \otimes\left(\Sigma_{m} / H \cdot\left(\partial \Delta_{+}^{k} \rightarrow \Delta_{+}^{k}\right)\right)
$$

for $m \geqslant 0$ and any $H \leqslant \Sigma_{m}$ (resp., $m \geqslant 1$ and any $H \leqslant \Sigma_{m}$ );

- weak equivalences are the stable equivalences of spectra.

Remark 2.15. Our terminology follows $[\mathbf{9}, \mathbf{1 6}]$ rather than $[\mathbf{1 4}, \mathbf{3}]$ which refer to $S$ cofibrations as "flat cofibrations". However, we make no explicit use of the maps referred to in $[\mathbf{9}, \mathbf{1 6}, \mathbf{1 4}, \mathbf{3}]$ simply as "cofibrations", even though our results also apply to those given that they are a subclass of $S$ cofibrations.

Remark 2.16. The proof of Proposition 3.13 shows that a $S$ cofibration $A \rightarrow B$ is also a positive $S$ cofibration iff $A_{0} \stackrel{\simeq}{\rightarrow} B_{0}$ is an isomorphism. We hence use positivity as a hypothesis in our results only if necessary and never as a conclusion, leaving it to the curious reader to check by direct calculation if positivity conclusions hold.

\subsection{Injective and projective model structures}

Definition 2.17. Let $\mathcal{C}$ be a model category, $M$ a monad on $\mathcal{C}$ and $\operatorname{Alg}_{M}$ the category of algebras over $M$.

The injective model structure on $\mathrm{Alg}_{M}$, if it exists, has as cofibrations (resp., weak equivalences) the underlying cofibrations (resp., weak equivalences) in $\mathcal{C}$.

The projective model structure on $\mathrm{Alg}_{M}$, if it exists, has as fibrations (resp., weak equivalences) the underlying fibrations (resp., weak equivalences) in $\mathcal{C}$.

Remark 2.18. Since most usual model structures are cofibrantly generated, it is often easier to build projective structures (cf. [15, Lemma 2.3]) than injective ones.

Remark 2.19. When in the presence of two monads, building injective structures does not in general commute with building projective structures.

A key example is given by comparing what we call the $\Sigma_{m}$-inj $G$-proj model structure on $\mathrm{S}_{*}^{G \times \Sigma_{m}}$, built as the $\Sigma_{m}$-injective structure over the $G$-projective structure over the standard model structure on $S_{*}$, with the $G$-proj $\Sigma_{m}$-inj model structure on $\mathrm{S}_{*}^{G \times \Sigma_{m}}$, which reverses the two constructions.

The former is shown to exist in Proposition 3.3, and examining the generating cofibrations, listed when proving Proposition 3.1, yields that cofibrations are those monomorphisms $A \hookrightarrow B$ adding only $G$-free simplices. Conversely, the latter is built by replacing the condition $H \cap G \times *=*$ in Proposition 3.1 with $H \subset * \times \Sigma_{m}$, so that cofibrations are those monomorphisms adding only simplices with such $H$ as isotropies. 
The need to distinguish between these two types of cofibration was first discovered by Pavlov and Scholbach and pointed to the author by Harper (see [4] for a discussion). In short, the fact that for $X_{m} \in \mathrm{S}_{*}^{\Sigma_{m}} \subset \mathrm{S}_{*}^{\Sigma}, m \geqslant 1$, then $\left(X_{m}\right)^{\otimes n} \in \mathrm{S}_{*}^{\Sigma_{m n} \times \Sigma_{n}} \subset$ $\mathrm{S}_{*}^{\Sigma \times \Sigma_{n}}$ is only " $\Sigma_{n}$-proj" cofibrant in the first sense (compare this with the proof of Theorem 1.2) is the key motivation for the $S \Sigma$-inj $\Sigma_{n}$-proj stable model structure on $\left(\mathrm{Sp}^{\Sigma}\right)^{\Sigma_{n}}$ introduced in this paper.

Remark 2.20. Remark 2.18 notwithstanding, we will often produce and iterate injective and projective structures. In fact, cofibrations in any of our model structures are obtained by iterating such constructions, and the interested reader will find we often choose names accordingly. As a typical example, the $S \Sigma$-inj $G$-proj cofibrations in $\left(\mathrm{Sp}^{\Sigma}\right)^{G}$ of Theorems 1.2 and 1.3 can be built by building a $S$-projective structure (abbreviated to $S$ following $[\mathbf{9}, \mathbf{1 6}]$ ) over a $\Sigma$-injective structure over a $G$-projective structure over the standard model structure in $S_{*}^{\mathbb{N}}$.

\subsection{Pushout product and left Quillen bifunctors}

Definition 2.21. Consider a bifunctor $\otimes$ from categories $\mathcal{C}, \mathcal{D}$ to a category $\mathcal{E}$, i.e., a functor of the form

$$
\mathcal{C} \times \mathcal{D} \stackrel{-\otimes-}{\longrightarrow} \mathcal{E}
$$

Given maps $c \stackrel{f}{\rightarrow} \bar{c}$ in $\mathcal{C}$ and $d \stackrel{g}{\rightarrow} \bar{d}$ in $\mathcal{D}$, we define their pushout product $f \square g$ (sometimes denoted $f \square^{\otimes} g$ to avoid confusion) to be the induced map

$$
c \otimes \bar{d} \underset{c \otimes d}{\amalg} \bar{c} \otimes d \stackrel{f \square g}{\longrightarrow} \bar{c} \otimes \bar{d} .
$$

For model categories $\mathcal{C}, \mathcal{D}, \mathcal{E}$ one defines the following (cf. [8, Def. 4.2.1]).

Definition 2.22. A bifunctor $\mathcal{C} \times \mathcal{D} \stackrel{-\otimes-}{\longrightarrow} \mathcal{E}$ between model categories is called a left Quillen bifunctor if

- for $c \in \mathcal{C}$ (resp., $d \in \mathcal{D})$, the functors $c \otimes(-): \mathcal{D} \rightarrow \mathcal{E}$ (resp., $(-) \otimes d: \mathcal{C} \rightarrow \mathcal{E}$ ) have right adjoints;

- $\otimes$ satisfies the pushout product axiom: for $f$ a cofibration in $\mathcal{C}$ and $g$ a cofibration in $\mathcal{D}, f \square g$ is a cofibration in $\mathcal{E}$, which is trivial if $f$ or $g$ is.

Remark 2.23. If $\mathcal{C}, \mathcal{D}$ are cofibrantly generated, a standard "retract of a transfinite composition of pushouts" argument (cf. [8, Lemma 4.2.4]) shows that it suffices to check the pushout product axiom for generating (trivial) cofibrations.

Remark 2.24. It is immediate that if $\otimes$ is a left Quillen bifunctor then both: (i) $c \otimes(-)$, $(-) \otimes d$ are left Quillen for cofibrant $c \in \mathcal{C}, d \in \mathcal{D}$; (ii) for $f$ in $\mathcal{C}$ and $g$ in $\mathcal{D}$ cofibrations between cofibrant objects, then so is $f \otimes g$.

However, as Remark 2.23 shows, it is technically preferable to verify the pushout product axiom rather than (i) or (ii). Indeed, that axiom is required to argue (i) via a filtration of $c, d$ and the analogue of Remark 2.23 fails for (ii).

\section{Model structures on $G$-spectra}

In this section we build the model structures featured in Theorems 1.2 and 1.3. 
Sections 3.1 and 3.2 build the $S \Sigma$-inj $G$-proj model structure on $\left(\mathrm{Sp}^{\Sigma}\right)^{G}$, the new "lax $G$-projective" structure capturing (for $G=\Sigma_{n}$ ) the $\Sigma_{n}$-cofibrancy of $X^{\wedge n}$ when $X$ is positive cofibrant. We closely follow the four model structures approach of $[\mathbf{1 6}]$, Section 3.1 dealing with $\mathrm{S}_{*}^{G \times \Sigma_{m}}$ and Section 3.2 with $\left(\mathrm{Sp}^{\Sigma}\right)^{G}$.

Section 3.3 builds the auxiliary monomorphism stable and $S$ stable model structures on $\left(\mathrm{Sp}^{\Sigma}\right)^{G}$ that appear in Theorem 1.3 and which, while technically novel, are just injective versions of the eponymous structures on $\mathrm{Sp}^{\Sigma}$ (cf. [9]).

\section{1. $\quad \Sigma$-inj $G$-proj model structure on $\mathrm{S}_{*}^{G \times \Sigma_{m}}$}

The following is well known. Remark 2.19 discusses the condition on $H$.

Proposition 3.1. For $G$ any finite group there exists a cofibrantly generated model structure on $\mathrm{S}_{*}^{G \times \Sigma_{m}}$ such that weak equivalences (resp., fibrations) are the maps $A \rightarrow B$ such that $A^{H} \rightarrow B^{H}$ is a weak equivalence (resp., fibration) in $\mathrm{S}_{*}$ for any $H \leqslant G \times \Sigma_{m}$ satisfying $H \cap G \times *=*$. Further, this is a left proper cellular simplicial model category.

Proof. We apply the usual small object argument in [8, Thm. 2.1.19] with the generating sets $I, J$ built from those in $S_{*}$ by inducing along each $H$. Explicitly,

$$
\begin{aligned}
& I=\bigcup_{H \cap G \times *=*}\left\{\left(G \times \Sigma_{m}\right) / H \cdot\left(\partial \Delta_{+}^{k} \rightarrow \Delta_{+}^{k}\right)\right\}, \\
& J=\bigcup_{H \cap G \times *=*}\left\{\left(G \times \Sigma_{m}\right) / H \cdot\left(\Lambda_{l+}^{k} \rightarrow \Delta_{+}^{k}\right)\right\} .
\end{aligned}
$$

Only the claim that maps in $J$-cell are weak equivalences is non-obvious. This follows for maps in $J$ by direct calculation, for pushouts of those by Proposition 2.3 and for transfinite compositions since those commute with $(-)^{H}$. Left properness, cellularity, and the simplicial model structure axioms are clear.

Remark 3.2. Analogous model structures can be built using more general conditions on $H$, such as families of subgroups.

Proposition 3.3. For $G$ any finite group there exists a cofibrantly generated model structure on $\mathrm{S}_{*}^{G \times \Sigma_{m}}$, which we call the $\Sigma_{m}$-inj G-proj model structure, where

- cofibrations are as in the model structure in Proposition 3.1;

- weak equivalences are the underlying weak equivalences in $\mathrm{S}_{*}$.

Further, this is a left proper cellular simplicial model category.

Proof. This follows by left Bousfield localization using [7, Thm. 4.1.1] with respect to a suitably chosen set of maps $\mathcal{S}$. We set (cf. [16, Prop. 1.3])

$$
\mathcal{S}=\left\{G \times \Sigma_{m} \cdot H E H_{+} \rightarrow\left(\left(G \times \Sigma_{m}\right) / H\right)_{+}: H \cap G \times *=*\right\},
$$

where $E H$ denotes a simplicial classifying space for $H$. It remains to show that the $\mathcal{S}$-equivalences are precisely the underlying weak equivalences.

Since the maps in $\mathcal{S}$ are underlying weak equivalences between cofibrant objects, [ 7 , Prop. 3.3.18(1)] applied to the forget-free power adjunction

$$
\text { fgt: } \mathrm{S}_{*}^{G \times \Sigma_{m}} \rightleftarrows \mathrm{S}_{*}:(-)^{\times\left(G \times \Sigma_{m}\right)}
$$

yields that all $\mathcal{S}$-local equivalences are underlying weak equivalences. 
To prove the converse it suffices to show that between $\mathcal{S}$-local objects any levelwise weak equivalence is a weak equivalence in the sense of Proposition 3.1. Since a fibrant object $X$ is $\mathcal{S}$-local precisely if one has induced weak equivalences

$$
X^{H}=\operatorname{Map}\left(\left(G \times \Sigma_{m} / H\right)_{+}, X\right)^{G \times \Sigma_{m}} \stackrel{\sim}{\rightarrow} \operatorname{Map}\left(G \times \Sigma_{m} \cdot H E H_{+}, X\right)^{G \times \Sigma_{m}}=X^{h H},
$$

the result follows due to $(-)^{h H}$ preserving underlying weak equivalences.

\subsection{Existence of the $S \Sigma$-inj $G$-proj stable model structure}

As in [16, Prop. 2.2], Proposition 3.3 induces a level model structure on $\left(\mathrm{Sp}^{\Sigma}\right)^{G}$.

Proposition 3.4. For $G$ any finite group there exists a cofibrantly generated model structure on $\left(\mathrm{Sp}^{\Sigma}\right)^{G}$, which we call the $S \Sigma$-inj G-proj level model structure, where

- weak equivalences are the maps $X \rightarrow Y$ such that $X_{m} \rightarrow Y_{m}, m \geqslant 0$ are underlying weak equivalences in $\mathrm{S}_{*}$.

- fibrations are the maps $X \rightarrow Y$ such that $X_{m} \rightarrow Y_{m}, m \geqslant 0$ are underlying fibrations in the $\Sigma_{m}$-inj G-proj model structure on $\mathrm{S}_{*}^{G \times \Sigma_{m}}$.

Further, this is a left proper cellular simplicial model category.

Proof. Let $I_{m}$ (resp., $J_{m}$ ) denote the sets of generating (resp., trivial) cofibrations for each $\Sigma_{m}$-inj $G$-proj model structure regarded as maps in $S_{*}^{G \times \Sigma}$. The proof of the existence of the model structure follows exactly as in [16, Prop. 2.2] by setting $I=\bigcup_{m \geqslant 0} S \otimes I_{m}$ (resp., $J=\bigcup_{m \geqslant 0} S \otimes J_{m}$ ) as the set of generating (resp., trivial) cofibrations in $\left(\mathrm{Sp}^{\Sigma}\right)^{G}$. The claims of left properness, cellularity, and the simplicial model structure axioms are again straightforward.

Remark 3.5. Analyzing the proofs of Propositions 3.1 and 3.4 yields an explicit description of the generating cofibrations in Proposition 3.4 (and Theorem 3.6)

$$
I=\left\{S \otimes\left(\left(G \times \Sigma_{m}\right) / H \cdot\left(\partial \Delta_{+}^{k} \rightarrow \Delta_{+}^{k}\right)\right): m \geqslant 0, H \cap G \times *=*\right\} .
$$

Theorem 3.6. For $G$ any finite group there exists a cofibrantly generated model structure on $\left(\mathrm{Sp}^{\Sigma}\right)^{G}$, which we call the $S \Sigma$-inj G-proj stable model structure, where

- cofibrations are as in the model structure in Proposition 3.4;

- weak equivalences are the underlying stable equivalences in $\mathrm{Sp}^{\Sigma}$.

Further, this is a left proper cellular simplicial model category.

Proof. This again follows by left Bousfield localization using [7, Thm. 4.1.1], this time localizing with respect to the set (cf. [16, Thm. 2.4])

$$
\mathcal{S}_{G}=\left\{S \otimes\left(G \times \Sigma_{m+1} \cdot S^{1} \rightarrow G \times \Sigma_{m} \cdot S^{0}\right): m \geqslant 0\right\} .
$$

It remains to check that $\mathcal{S}_{G}$-equivalences coincide with stable equivalences.

For $G=*$ this is well known since then our model structure reduces to that of $[\mathbf{1 6}$, Thm. 2.4].

We will reduce the general case to the case $G=*$. To do so, start by considering the $S G$-proj $\Sigma$-inj stable model structure on $\left(\mathrm{Sp}^{\Sigma}\right)^{G}$, which is built as the $G$-projective 
model structure over the model structure on $\mathrm{Sp}^{\Sigma}$ in the previous paragraph. We claim this model structure can alternatively be built by first building its level version, then localizing with respect to $\mathcal{S}_{G}$. Since both procedures create localizations of said level version, it suffices to check that they lead to the same local objects, and that follows since $\mathcal{S}_{G}=G \cdot \mathcal{S}_{*}$.

To relate this to our intended model structure, consider the identity Quillen equivalence

$$
\mathrm{Id}:\left(\mathrm{Sp}^{\Sigma}\right)^{G} \rightleftarrows\left(\mathrm{Sp}^{\Sigma}\right)^{G}: \mathrm{Id},
$$

where the left hand $\left(\mathrm{Sp}^{\Sigma}\right)^{G}$ has the $S G$-proj $\Sigma$-inj level model structure and the right hand $\left(\mathrm{Sp}^{\Sigma}\right)^{G}$ has the $S \Sigma$-inj $G$-proj level model structure. It now suffices to check both sides have the same $\mathcal{S}_{G}$-local equivalences, and this is clear since mapping spaces can be simultaneously computed using cofibrant replacements in the left hand side and fibrant replacements in the right hand side.

\subsection{Monomorphism stable and $S$ stable model structures}

When $G=*$, the existence of the following model structure is asserted without proof in the discussion preceding [9, Thm. 5.3.7]. For the sake of completeness (and as a warm-up to Theorem 5.46), we include a proof sketch combining arguments of [9, Sec. 5] with the localization machinery of [7, Thm. 4.1.1].

Theorem 3.7. For $G$ any finite group there exists a cofibrantly generated model structure on $\left(\mathrm{Sp}^{\Sigma}\right)^{G}$, which we call the monomorphism stable model structure, where

- cofibrations are the maps $X \rightarrow Y$ such that $X_{m} \rightarrow Y_{m}$ is a monomorphism of pointed simplicial sets for each $m \geqslant 0$;

- weak equivalences are the underlying stable equivalences in $\mathrm{Sp}^{\Sigma}$.

Further, this is a left proper cellular simplicial model category.

Proof. We start by building the analogue level weak equivalence model structure. When $G=*$, this is precisely the injective level structure in [9, Thm. 5.1.2], and the interested reader can check that the somewhat lengthy proof there generalizes. Instead, we point out that much of the argument can be streamlined by instead verifying the conditions in [8, Thm. 2.1.19].

Setting $I$ (resp., $J$ ) to be a set of representatives of monomorphisms (resp., monomorphisms that are level weak equivalences) between countable $G$-spectra (cf. proof of [9, Thm. 5.1.2]), parts 1, 2, 3 of [8, Thm. 2.1.19] are immediate, part 4 follows since $J \subset I$ and colimits are levelwise and part 5 follows by noting that $I$ contains the maps of the form $S \otimes\left(G \times \Sigma_{m} \cdot\left(\partial \Delta_{+}^{k} \rightarrow \Delta_{+}^{k}\right)\right)$, so that $I$-inj consists of level equivalences. For the harder part 6 , one needs to show a lift exists in any diagram

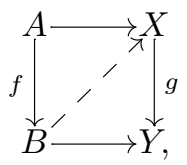

with $f \in \mathcal{W} \cap I$-cof and $g \in J$-inj. This generalizes [9, Lemma 5.14(6)], the proof of which applies without change once one generalizes [9, Lemma 5.17] to $G$-spectra. 
The latter can be done by simply choosing the $F C$ subspectra in the proof of $[\mathbf{9}$, Lemma 5.17] to be $G$-subspectra, finishing the existence argument for the level model structure. Left properness, cellularity and the simplicial model structure axioms are again straightforward.

To produce the stable version, one again applies [7, Thm. 4.1.1] to the set $\mathcal{S}_{G}$ in the proof of Theorem 3.6, showing that the weak equivalences are as described by arguing as in the last paragraph of the proof of that theorem.

Theorem 3.8. For $G$ any finite group there exists a cofibrantly generated model structure on $\left(\mathrm{Sp}^{\Sigma}\right)^{G}$, which we call the $S$ stable model structure, where

- a set of generating cofibrations is

$$
I=\left\{S \otimes\left(\left(G \times \Sigma_{m}\right) / H \cdot\left(\partial \Delta_{+}^{k} \rightarrow \Delta_{+}^{k}\right)\right): m \geqslant 0, \text { any } H \leqslant G \times \Sigma_{m}\right\} ;
$$

- weak equivalences are the underlying stable equivalences in $\mathrm{Sp}^{\Sigma}$.

Further, this is a left proper cellular simplicial model category.

Proof. This is an analogue of Theorem 3.6, now without conditions on $H$. The same proof, starting with analogues of Propositions 3.1, 3.3 and 3.4, applies.

Proposition 3.9. The $S$ stable model structure on $\left(\mathrm{Sp}^{\Sigma}\right)^{G}$ is the injective model structure over the $S$ stable model structure on $\mathrm{Sp}^{\Sigma}$.

More explicitly, the $S$ stable cofibrations (resp., weak equivalences) in $\left(\mathrm{Sp}^{\Sigma}\right)^{G}$ are the underlying $S$ stable cofibrations (resp., weak equivalences) in $\mathrm{Sp}^{\Sigma}$.

To prove Proposition 3.9 we start by recalling a well known inductive procedure to build maps of spectra (cf. [14, II.5], [9, Sec. 5.2]).

Definition 3.10. Define $\bar{S} \in \mathrm{Sp}^{\Sigma}$ by $\bar{S}_{0}=*, \bar{S}_{m}=S^{m}$ together with the obvious structure maps and let $i: \bar{S} \rightarrow S$ be the inclusion. For $A \in \mathrm{Sp}^{\Sigma}$, define its $m$-th latching object to be $L_{m} A=(\bar{S} \wedge A)_{m}$ for $m \geqslant 0$. Note that $i$ induces a $m$-th latching map

$$
L_{m} A \stackrel{l_{m} A}{\longrightarrow} A_{m} .
$$

Given spectra $A, B$ define a map up to degree $m$ from $A$ to $B$ to be a list of maps $\left\{f_{\bar{m}}: A_{\bar{m}} \rightarrow B_{\bar{m}}\right\}_{0 \leqslant \bar{m} \leqslant m}$ compatible with the spectra structure maps up to degree $m$. The importance of latching maps comes from the following result (used implicitly in $[\mathbf{9}$, Sec. 5.2.2]. Also, compare with [13, Obs. 3.9]).

Lemma 3.11. A map $\left\{f_{\bar{m}}: A_{\bar{m}} \rightarrow B_{\bar{m}}\right\}_{0 \leqslant \bar{m} \leqslant m-1}$ up to degree $m-1$ naturally induces a map $L_{m} A \rightarrow L_{m} B$. Further, extensions to a map $\left\{f_{\bar{m}}: A_{\bar{m}} \rightarrow B_{\bar{m}}\right\}_{0 \leqslant \bar{m} \leqslant m}$ up to degree $m$ are in natural bijection with dashed arrows

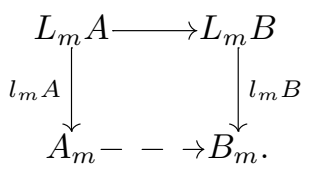

Remark 3.12. By naturality Lemma 3.11 generalizes to $\left(\mathrm{Sp}^{\Sigma}\right)^{G}$.

Proposition 3.9 will follow from the following analogue of [9, Sec. 5.2.2]. 
Proposition 3.13. The $S$ stable cofibrations in $\left(\mathrm{Sp}^{\Sigma}\right)^{G}$ are those maps $f: A \rightarrow B$ such that $(f \square i)_{m}: A_{m} \vee_{L_{m} A} L_{m} B \rightarrow B_{m}$ is a monomorphism for all $m \geqslant 0$.

Proof. $X \rightarrow Y$ is a $S$ stable trivial fibration iff $X_{m}^{H} \rightarrow Y_{m}^{H}$ are trivial fibrations in $\mathrm{S}_{*}$ for all $m \geqslant 0, H \leqslant G \times \Sigma_{m}$, i.e., iff $X_{m} \rightarrow Y_{m}$ are genuine $G \times \Sigma_{m}$ fibrations for $m \geqslant 0$. By Lemma 3.11, building a lift in the left hand diagram
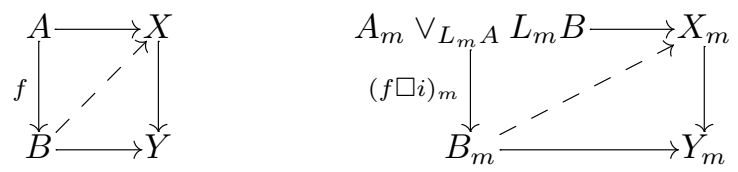

is the same as building successive lifts in the right hand diagrams for $m \geqslant 0$. Since monomorphisms have the left lifting property against genuine fibrations, the given condition is sufficient.

For the converse, by $\left[\mathbf{8}\right.$, Lemma 4.2.4] it suffices to check $(f \square i)_{m}$ is a monomorphism when $f$ is a generating cofibration. Letting

$$
f=S \otimes\left(\left(G \times \Sigma_{m}\right) / H \cdot \partial \Delta_{+}^{k} \stackrel{f^{\prime}}{\rightarrow}\left(G \times \Sigma_{m}\right) / H \cdot \Delta_{+}^{k}\right)
$$

one has $f \square i=f^{\prime} \square^{\otimes} i$ (where $\square^{\otimes}$ denotes the pushout product with respect to the bifunctor $\mathrm{S}_{*}^{\Sigma} \times \mathrm{Sp}^{\Sigma} \stackrel{\otimes}{\longrightarrow} \mathrm{Sp}^{\Sigma}$ ) so that the result is now clear.

Proof of Proposition 3.9. Cofibrations are underlying since forgetting the $G$-action does not change the characterization in Proposition 3.13. The case of weak equivalences is obvious.

Remark 3.14. In Section 5.3 we will use the $\Sigma_{r}$-injective model structure on $\left(\mathrm{Sp}^{\Sigma}\right)^{G \times \Sigma_{r}}$ with regard to the $S \Sigma$-inj $G$-proj model structure on $\left(\mathrm{Sp}^{\Sigma}\right)^{G}$. We call this the $S$ $\Sigma \times \Sigma_{r}$-inj $G$-proj stable model structure, and build it just as in Theorems 3.6 and 3.8 using as generating cofibrations

$$
I=\left\{S \otimes\left(\left(G \times \Sigma_{m} \times \Sigma_{r}\right) / H \cdot\left(\partial \Delta_{+}^{k} \rightarrow \Delta_{+}^{k}\right)\right): m, r \geqslant 0, H \cap G \times * \times *=*\right\} .
$$

The analogue of Proposition 3.9 proving $\Sigma_{r}$-injectiveness is shown in the same way by noting that $X \rightarrow Y$ is a trivial fibration iff $X_{m}^{H} \rightarrow Y_{m}^{H}, H \cap G \times * \times *=*$ is a trivial fibration, so that $A \rightarrow B$ is a cofibration iff $(f \square i)_{m}: A_{m} \vee_{L_{m} A} L_{m} B \rightarrow B_{m}$ is built only out of simplices with isotropies $H$ satisfying $H \cap G \times * \times *=*$.

\section{Properties of $S \sum$-inj $G$-proj cofibrations}

In this section we prove the key properties of $S \Sigma$-inj $G$-proj cofibrations.

Subsection 4.1 deals with those properties one would expect from genuine $G$-projective cofibrations, namely the "change of group" Propositions 4.1 and 4.2 as well as Theorem 1.3.

Subsection 4.2, the technical heart of the paper, deals with the somewhat lengthier proof of Theorem 1.2. 
COFIBRANCY OF OPERADIC CONSTRUCTIONS IN POSITIVE SYMMETRIC SPECTRA 147

\section{1. $\quad G$-projective type properties}

Proposition 4.1. Suppose each category is equipped with its respective $S \Sigma$-inj $G$ proj stable model structure. Then the functor

$$
\left(\mathrm{Sp}^{\Sigma}\right)^{G} \times\left(\mathrm{Sp}^{\Sigma}\right)^{\bar{G}} \stackrel{-\wedge-}{\longrightarrow}\left(\mathrm{Sp}^{\Sigma}\right)^{G \times \bar{G}}
$$

is a left Quillen bifunctor.

Proof. The existence of right adjoints is formal. It suffices to check the pushout product axiom (cf. Definition 2.22) between generating (trivial) cofibrations (cf. Remark 2.23) and letting (cf. Remark 3.5)

$$
f=S \otimes\left(\left(G \times \Sigma_{m}\right) / H \cdot\left(\partial \Delta_{+}^{k} \rightarrow \Delta_{+}^{k}\right)\right), \quad g=S \otimes\left(\left(\bar{G} \times \Sigma_{\bar{m}}\right) / \bar{H} \cdot\left(\partial \Delta_{+}^{\bar{k}} \rightarrow \Delta_{+}^{\bar{k}}\right)\right)
$$

one has (using the identification $H \times \bar{H} \subset G \times \Sigma_{m} \times \bar{G} \times \Sigma_{\bar{m}} \subset G \times \bar{G} \times \Sigma_{m+\bar{m}}$ )

$$
f \square g=S \otimes\left(\left(G \times \bar{G} \times \Sigma_{m+\bar{m}}\right) /(H \times \bar{H}) \cdot\left(\left(\partial\left(\Delta^{k} \times \Delta^{\bar{k}}\right)\right)_{+} \rightarrow\left(\Delta^{k} \times \Delta^{\bar{k}}\right)_{+}\right)\right),
$$

which is a cofibration since Remark 3.5 implies $H \times \bar{H} \cap G \times \bar{G} \times\{*\}=*$.

The extra claim that $f \square g$ is a weak equivalence if either $f$ or $g$ is can be checked by forgetting the actions of $G, \bar{G}$, reducing to $[\mathbf{9}$, Thm. 5.3.7(5)].

Proposition 4.2. Let $\bar{G} \subset G$ be finite groups, and suppose each category is equipped with its respective $S \Sigma$-inj $G$-proj stable model structure. Then both

$$
\text { fgt: }\left(\mathrm{Sp}^{\Sigma}\right)^{G} \rightleftarrows\left(\mathrm{Sp}^{\Sigma}\right)^{\bar{G}}:\left((-)^{G \cdot S}\right)^{\bar{G}} \quad \text { and } \quad G \cdot \bar{G}(-):\left(\mathrm{Sp}^{\Sigma}\right)^{\bar{G}} \rightleftarrows\left(\mathrm{Sp}^{\Sigma}\right)^{G}: \mathrm{fgt}
$$

are Quillen adjunctions.

Proof. This is immediate for the first adjunction since fgt preserves weak equivalences and free actions. For the second one, choose a generating cofibration

$$
f=S \otimes\left(\left(\bar{G} \times \Sigma_{m}\right) / H \cdot\left(\partial \Delta_{+}^{k} \rightarrow \Delta_{+}^{k}\right)\right)
$$

so that

$$
G \cdot{ }_{\bar{G}} f=S \otimes\left(\left(G \times \Sigma_{m}\right) / H \cdot\left(\partial \Delta_{+}^{k} \rightarrow \Delta_{+}^{k}\right)\right),
$$

which is again a cofibration since $H \cap \bar{G} \times\{*\}=*$ implies $H \cap G \times\{*\}=*$.

That $G \cdot{ }_{\bar{G}}(-)$ applied to a trivial cofibration yields a weak equivalence follows by forgetting the actions since then $G \cdot{ }_{\bar{G}}(-)$ is a wedge over $G / \bar{G}$.

We now turn to the proof of Theorem 1.3. We will make use of the following analogue for bifunctors of the "universal property of left Bousfield localizations" in [7, Prop. 3.3.18(1)].

Lemma 4.3. Suppose

$$
\mathcal{C} \times \mathcal{D} \stackrel{-\otimes-}{\longrightarrow} \mathcal{E}
$$

is a left Quillen bifunctor, that $\mathcal{S}$ is a class of maps between cofibrant objects of $\mathcal{C}$ such that the left Bousfield localization $L_{\mathcal{S}} \mathcal{C}$ exists, and that $\mathcal{D}$ is a cofibrantly 
generated model category for which the generating cofibrations have cofibrant domains and codomains. Then (recall that as categories $L_{\mathcal{S}} \mathcal{C}=\mathcal{C}$ )

$$
L_{\mathcal{S}} \mathcal{C} \times \mathcal{D} \stackrel{-\otimes-}{\longrightarrow} \mathcal{E}
$$

remains a left Quillen bifunctor iff $f \otimes d$ is a weak equivalence in $\mathcal{E}$ for each $f \in \mathcal{S}$ and $d$ a domain or codomain of a generating cofibration of $\mathcal{D}$.

Proof. First note that by Remark $2.23 \otimes$ will remain a left Quillen bifunctor precisely if the pushout product axiom holds when $f: c \rightarrow \bar{c}$ is a trivial cofibration in $L_{\mathcal{S}} \mathcal{C}$ and $g: d \rightarrow \bar{d}$ is a generating cofibration in $\mathcal{D}$. Since $(-) \otimes d,(-) \otimes \bar{d}$ are left Quillen functors with respect to the original model structure $\mathcal{C}$, [7, Prop. 3.3.18(1)] shows that the condition in the theorem is necessary and that, if that condition holds, the horizontal maps in

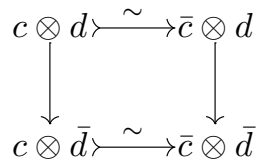

are trivial cofibrations. The 2-out-of-3 property now implies that $f \square g$ is a weak equivalence, showing that the condition in the theorem is also sufficient.

Proof of Theorem 1.3. The existence of the required right adjoints is formal.

We will prove the remainder of both parts in parallel.

As a first step we prove the analogue result with stable structures replaced by level structures throughout. Since the generating (trivial) cofibrations in the $S \Sigma$-inj $G$-proj level model structure all have the form $S \otimes f$ for some $f$ in $\left(\mathrm{S}_{*}\right)^{G}$ (cf. Remark 3.5), this reduces to showing the analogue result for the bifunctor

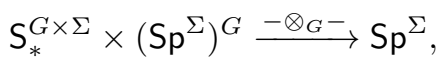

where $\mathrm{S}_{*}^{G \times \Sigma}$ has the $\Sigma$-inj $G$-proj model structure obtained by combining the $\Sigma_{m}$-inj $G$-proj model structures of Proposition 3.3 for each $m \geqslant 0$.

For the monomorphism case, choose (cf. Proposition 3.1) a generating (resp., trivial) cofibration in $\mathrm{S}_{*}^{G \times \Sigma}$

$$
f=\left(G \times \Sigma_{m}\right) / H \cdot f^{\prime}
$$

( $f^{\prime}$ a generating (resp., trivial) cofibration in $\mathrm{S}_{*}, H \cap G \times *=*$ ) and a monomorphism $g$ in $\left(S p^{\Sigma}\right)^{G}$. Then, using the identification $\Sigma_{m} \times \Sigma_{\bar{m}-m} \subset \Sigma_{\bar{m}}$,

$$
\begin{aligned}
\left(f \square^{\otimes G} g\right)_{\bar{m}} & \simeq\left(\left(f \square^{\otimes} g\right)_{\bar{m}}\right)_{G} \simeq\left(G \times \Sigma_{\bar{m}} \underset{H \times \Sigma_{\bar{m}-m}}{ } f^{\prime} \square^{\wedge} g_{\bar{m}-m}\right)_{G} \simeq \\
& \simeq\left(G \backslash\left(G \times \Sigma_{\bar{m}}\right) /\left(H \times \Sigma_{\bar{m}-m}\right)\right) \cdot f^{\prime} \square^{\wedge} g_{\bar{m}-m},
\end{aligned}
$$

where the last step follows since the condition $H \cap G \times *=*$ implies $G$ acts freely on cosets $\left(G \times \Sigma_{\bar{m}}\right) /\left(H \times \Sigma_{\bar{m}-m}\right)$. It is now clear that $f \square^{\otimes_{G}} g$ is a monomorphism, level trivial if either $f^{\prime}$ or all $g_{m}$ are.

For the $S$ level case, note first that by the monomorphism case we need no longer worry about trivial cofibrations. For the case of regular cofibrations, choose generating 
cofibrations $f$ in $\mathrm{S}_{*}^{G \times \Sigma}$ as above and

$$
g=S \otimes\left(\left(G \times \Sigma_{\bar{m}}\right) / \bar{H} \cdot g^{\prime}\right)
$$

( $g^{\prime}$ a generating cofibration in $\mathrm{S}_{*}$, any $\left.\bar{H} \leqslant G \times \Sigma_{\bar{m}}\right)$ in $\left(\mathrm{Sp}^{\Sigma}\right)^{G}$. Then, using the identification $\Sigma_{m} \times \Sigma_{\bar{m}} \subset \Sigma_{m+\bar{m}}$,

$$
f \square^{\otimes G} g=\left(f \square^{\otimes} g\right)_{G}=S \otimes\left(G \backslash\left(G \times G \times \Sigma_{m+\bar{m}}\right) /(H \times \bar{H}) \cdot f^{\prime} \square g^{\prime}\right),
$$

which is indeed a $S$ cofibration, finishing the proof of the analogue level result.

We now turn to the second step, showing that $\wedge_{G}$ remains a left Quillen bifunctor after stabilizing the model structures. In all cases one is localizing by $\mathcal{S}_{G}$ (cf. proofs of Theorems $3.6,3.7,3.8$ ), and hence by Lemma 4.3 it suffices to verify $f \otimes_{G} A$ is a stable equivalence for $f \in \mathcal{S}_{G}$ and $A$ a suitably cofibrant $G$-spectrum. It suffices to deal with the case of monomorphism cofibrant $A$ (i.e., any $A$ ), and since $\mathcal{S}_{G}=G \cdot \mathcal{S}_{*}$ this reduces to the case $G=*$. But for $G=*$ the claim follows by [9, Thm. 5.3.7(5)], finishing the proof.

\subsection{Lax $\Sigma_{n}$-cofibrancy of $n$-fold pushout products}

In this section we prove Theorem 1.2. Roughly speaking, the proof will follow by induction using the usual "retract of a transfinite composition of pushouts of generating cofibrations" description of cofibrations. The main obstacle is the fact that the $n$-fold pushout product $\square^{n}$ does not respect compositions of maps. Handling those will require two key technical results, Lemmas 4.8 and 4.10 .

To prove Lemmas 4.8, 4.10 and Theorem 1.2 we will need some notation.

Definition 4.4. Let $I$ be a poset and $i: I \rightarrow \mathrm{Sp}^{\Sigma}$. We denote by $i^{\wedge n}$ the "cubical" diagram

$$
i^{\wedge n}: I^{\times n} \stackrel{i^{\times n}}{\longrightarrow}\left(\mathrm{Sp}^{\Sigma}\right)^{\times n} \stackrel{\wedge}{\longrightarrow} \mathrm{Sp}^{\Sigma} .
$$

Further, for $T \subset I^{\times n}$ any subset, we denote $Q_{T}^{n}(i)=\operatorname{colim}_{T}\left(i^{\wedge n}\right)$. Note that when $T$ is closed under the obvious $\Sigma_{n}$-action on $I^{\times n}$ one obtains an induced $\Sigma_{n}$-action on $Q_{T}^{n}(i)$.

Remark 4.5. Borrowing from [3], we let $Q_{t}^{n}(i)$ denote $Q_{T_{t}}^{n}(i)$, where $i=X \rightarrow Y$ is viewed as a functor $(0 \rightarrow 1) \rightarrow \mathrm{Sp}^{\Sigma}$ and $T_{t}$ is the subset of $(0 \rightarrow 1)^{\times n}$ of those tuples with at most $t$ 1-entries.

The objects $Q_{T}^{n}(i)$ are related to latching objects/maps (cf. [13, Obs. 3.8]).

Definition 4.6. Given $e \in I^{\times n}$ set $T_{e}^{n}=\left\{\bar{e} \in I^{\times n}: \bar{e}<e\right\}$. Further, given $i: I \rightarrow$ $\mathrm{Sp}^{\Sigma}$, define the latching map of $i^{\wedge n}$ at $e$ as the natural map

$$
L_{e}^{n}\left(i^{\wedge n}\right)=Q_{T_{e}}^{n}(i) \stackrel{l_{e}^{n}\left(i^{\wedge n}\right)}{\longrightarrow} i^{\wedge n}(e) .
$$

A straightforward computation reveals the following relationship between latching maps and the pushout product (cf. [13, Example 4.6]).

Proposition 4.7. Let $e_{1} \in I^{\times n_{1}}$ and $e_{2} \in I^{\times n_{2}}$, so that $\left(e_{1}, e_{2}\right) \in I^{\times\left(n_{1}+n_{2}\right)}$. Then

$$
l_{\left(e_{1}, e_{2}\right)}^{n_{1}+n_{2}}\left(i^{\wedge\left(n_{1}+n_{2}\right)}\right)=l_{e_{1}}^{n_{1}}\left(i^{\wedge n_{1}}\right) \square l_{e_{2}}^{n_{2}}\left(i^{\wedge n_{2}}\right) .
$$


The following is the key technical lemma in this section. The proof of this result, which is essentially lifted from the appendix to the author's thesis, ${ }^{2}$ explores generalizations of filtrations found in [1, Sec. 12], [3, Def. 4.13] from single maps to compositions of maps. A similar result, with modified hypotheses and conclusions but sharing some of the key ideas in the proof, was proven independently by David White in [17].

Lemma 4.8. Let $i:(0 \rightarrow 1 \rightarrow 2) \rightarrow \mathrm{Sp}^{\Sigma}$ be a diagram $Z_{0} \stackrel{f_{1}}{\longrightarrow} Z_{1} \stackrel{f_{2}}{\longrightarrow} Z_{2}$ such that

$$
f_{i}^{\square \bar{n}}: Q_{\bar{n}-1}^{\bar{n}}\left(f_{i}\right) \rightarrow Z_{i}^{\wedge \bar{n}}, \quad 0 \leqslant \bar{n} \leqslant n, i=1,2
$$

are $S \Sigma$-inj $\Sigma_{\bar{n}}$-proj cofibrations in $\left(\mathrm{Sp}^{\Sigma}\right)^{\Sigma_{\bar{n}}}$.

Choose $T \subset \bar{T} \subset(0 \rightarrow 1 \rightarrow 2)^{\times n}$ symmetric convex (recall $T$ is called convex if $e \in T$ and $\bar{e} \leqslant e$ implies $\bar{e} \in T$ ) subsets containing any tuple that has at least one 0 -entry. Then the map

$$
Q_{T}^{n}(i) \rightarrow Q_{T}^{n}(i)
$$

is a $S \Sigma$-inj $\Sigma_{n}$-proj stable cofibration.

Additionally, if one also knows that $Z_{0}^{\wedge \bar{n}}, 0 \leqslant \bar{n} \leqslant n$ is $S \Sigma$-inj $\Sigma_{\bar{n}}$-proj cofibrant then the conclusion above holds for any symmetric convex $T \subset \bar{T}$.

Proof. We deal with the main and additional cases in parallel.

Without loss of generality we assume $\bar{T}$ is obtained from $T$ by adding the orbit of some $e=\left(e_{0}, e_{1}, e_{2}\right) \in\{0\}^{\times n_{0}} \times\{1\}^{\times n_{1}} \times\{2\}^{\times n_{2}}$. Then $T_{e}^{n} \subset T$ and one has a pushout diagram

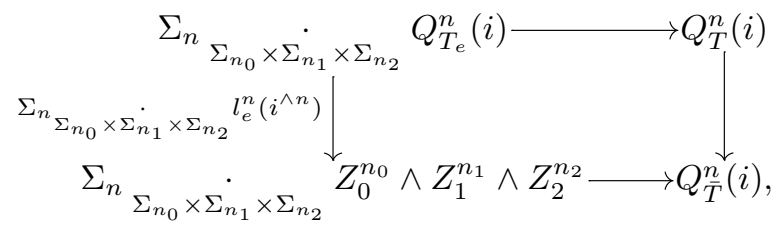

so that it suffices to show that the left hand map is a $S \Sigma$-inj $\Sigma_{n}$-proj cofibration, and by Proposition 4.2 this reduces to showing that the latching map $l_{e}^{n}\left(i^{\wedge n}\right)$ is a $S$ $\Sigma$-inj $\Sigma_{n_{0}} \times \Sigma_{n_{1}} \times \Sigma_{n_{2}}$-proj cofibration. Proposition 4.7 then identifies

$$
l_{e}^{n}\left(i^{\wedge n}\right)=l_{e_{0}}^{n_{0}}\left(i^{\wedge n_{0}}\right) \square l_{e_{1}}^{n_{1}}\left(i^{\wedge n_{1}}\right) \square l_{e_{2}}^{n_{2}}\left(i^{\wedge n_{2}}\right)=Z_{0}^{\wedge n_{0}} \wedge f_{1}^{\square n_{1}} \square f_{2}^{\square n_{2}}
$$

(for the identification $l_{e_{2}}^{n_{2}}\left(i^{\wedge n_{2}}\right)=f_{2}^{\square n_{2}}$, note that the tuples without 0-entries are final in $\left.T_{e_{2}}^{n_{2}} \subset(0 \rightarrow 1 \rightarrow 2)^{\times n_{2}}\right)$. Now note that in the main case $T$ already contains all tuples with a 0 -entry so that it must be $n_{0}=0$, while in the additional case $n_{0}$ can take any value. In either case Proposition 4.1 finishes the proof.

Remark 4.9. While it is straightforward to generalize Lemma 4.8 to longer compositions (of three or more maps), such generalizations will not be necessary.

Lemma 4.10. Let $Z_{0} \stackrel{f_{1}}{\longrightarrow} Z_{1} \stackrel{f_{2}}{\longrightarrow} Z_{2}$ be as in Lemma 4.8 .

${ }^{2}$ That appendix proved the analogous claim for $\Sigma_{n}$-projective cofibrations, an ultimately useless fact since the $\Sigma_{n}$-projective analogue of Theorem 1.2 fails for generating cofibrations. 
If one knows additionally that $Z_{0}^{\wedge \bar{n}}, 0 \leqslant \bar{n} \leqslant n$ is $S \Sigma_{\text {-inj }} \Sigma_{\bar{n}}$-proj cofibrant, then the maps (where the $Q_{\bar{n}}^{n}$ objects are defined in Remark 4.5)

$$
Q_{\bar{n}}^{n}\left(f_{2} f_{1}\right) \bigvee_{Q_{\bar{n}}^{n}\left(f_{1}\right)} Q_{\bar{n}+1}^{n}\left(f_{1}\right) \rightarrow Q_{\bar{n}+1}^{n}\left(f_{2} f_{1}\right), \quad 0 \leqslant \bar{n}<n
$$

are $S \Sigma$-inj $\Sigma_{n}$-proj stable cofibrations.

Further, absent the additional condition, the result still holds when $\bar{n}=n-1$.

Proof. This is a direct consequence of Lemma 4.8 by identifying all objects with $Q_{T}^{n}(i)$ for some $T$. For $Q_{k}^{n}\left(f_{1}\right)$ this is $T_{k}^{1}$, the subset of tuples with no 2 -entries and at most $k$ 1-entries, while for $Q_{k}^{n}\left(f_{2} f_{1}\right)$ it is $T_{k}^{2}$, the subset of tuples with at least $n-k$ 0 -entries (or equivalently, at most $k 2$-or-1-entries). The result then follows by noting that $T_{\bar{n}}^{2} \cap T_{\bar{n}+1}^{1}=T_{\bar{n}}^{1}$ and $T_{\bar{n}}^{2} \cup T_{\bar{n}+1}^{1} \subset T_{\bar{n}+1}^{2}$.

All we are now missing to prove Theorem 1.2 is the following lemma, which handles the pushout case (compare with [3, Prop. 6.13]).

Lemma 4.11. Consider a pushout diagram

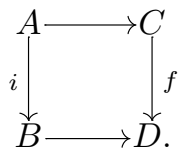

If $i^{\square n}$ is a (trivial) $S \Sigma$-inj $\Sigma_{n}$-proj cofibration in $\left(\mathrm{Sp}^{\Sigma}\right)^{\Sigma_{n}}$ then so is $f^{\square n}$.

Proof. It suffices to show that

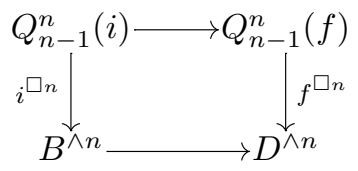

is itself a pushout diagram. This is [3, Prop. 6.13], where it is left as an exercise. Alternatively, note that the pushout product $\square$ is a bifunctor between arrow categories (cf. [13, Def. 4.4.]) which takes pushout diagrams in each arrow variable to pushout diagrams, so that the result follows by considering the arrow category diagram $i^{\square n} \rightarrow$ $f \square i^{\square(n-1)} \rightarrow f^{\square 2} \square i^{\square(n-2)} \rightarrow \cdots \rightarrow f^{\square n}$.

We now prove Theorem 1.2. The proof is similar to that of [4, Prop. 4.28*] (also, compare [3, Prop. 4.28]), except now boosted by Lemma 4.10.

Proof of Theorem 1.2. Since weak equivalences ignore the $\Sigma_{n}$-action and the $S$ stable model structure on $\mathrm{Sp}^{\Sigma}$ is monoidal (cf. [9, Thm. 5.5.1]), we need not worry about trivial cofibrations.

We argue by induction on a description of a positive $S$ cofibration $f$ in $\mathrm{Sp}^{\Sigma}$ as a retract of a transfinite composition of pushouts of generating cofibrations. 
The base case is that of a generating cofibration $f=S \otimes\left(\Sigma_{m} / H \cdot\left(\partial \Delta_{+}^{k} \rightarrow \Delta_{+}^{k}\right)\right)$ for some $m \geqslant 1, H \leqslant \Sigma_{m}$. Then, using the identifications $H^{\times n} \subset\left(\Sigma_{m}\right)^{\times n} \subset \Sigma_{m n}$,

$$
f^{\square n}=S \otimes\left(\Sigma_{m n} / H^{\times n} \cdot\left(\partial\left(\Delta^{k}\right)_{+}^{\times n} \rightarrow\left(\Delta^{k}\right)_{+}^{\times n}\right)\right),
$$

which is a $S \Sigma$-inj $\Sigma_{n}$-proj cofibration since the condition $m \geqslant 1$ implies the map

$$
\left(\Sigma_{m n} / H^{\times n} \cdot\left(\partial\left(\Delta^{k}\right)_{+}^{\times n} \rightarrow\left(\Delta^{k}\right)_{+}^{\times n}\right)\right)
$$

is built by adding only $\Sigma_{n}$-free simplices.

We now move to the general case. As usual, retracts cause no difficulty, and we hence focus on a transfinite composition

$$
A_{0} \stackrel{f_{0}}{\longrightarrow} A_{1} \stackrel{f_{1}}{\longrightarrow} A_{2} \stackrel{f_{2}}{\longrightarrow} A_{3} \stackrel{f_{3}}{\longrightarrow} \cdots \rightarrow A_{\kappa}=\operatorname{colim}_{\beta<\kappa} A_{\beta}
$$

(we use the convention $A_{\beta}=\operatorname{colim}_{\gamma<\beta} A_{\gamma}$ for each limit ordinal $\beta<\kappa$ ) where each $f_{\beta}: A_{\beta} \rightarrow A_{\beta+1}$ is the pushout of a generating positive $S$ cofibration $i_{\beta}$. Further, for $\beta \leqslant \kappa$, denote by $\bar{f}_{\beta}: A_{0} \rightarrow A_{\beta}$ the full composite of $\left\{f_{\gamma}\right\}_{\gamma<\beta}$. Since the $Q_{t}^{n}$ constructions preserve filtered colimits (since so does $\wedge$ in each variable), the main claim will follow if the vertical map of $\kappa$-diagrams ( $\kappa$-th map excluded)

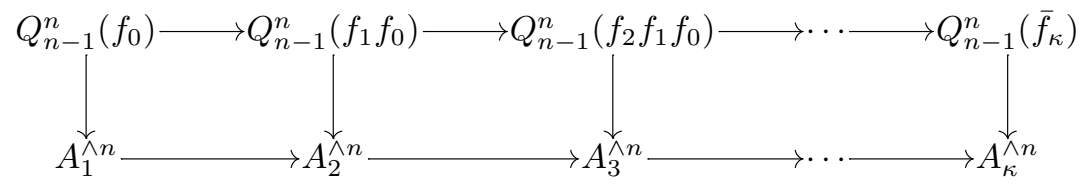

is a $\kappa$-projective cofibration between $\kappa$-diagrams with respect to the underlying $S \Sigma$ inj $\Sigma_{n}$-proj model structure. One thus reduces to inductively checking that the relative latching maps $A_{\beta}^{\wedge n} \vee_{Q_{n-1}^{n}\left(\bar{f}_{\beta}\right)} Q_{n-1}^{n}\left(f_{\beta} \bar{f}_{\beta}\right) \rightarrow A_{\beta+1}^{\wedge n}$ for successor ordinals $\beta+1$ are $S$ $\Sigma$-inj $\Sigma_{n}$-proj cofibrations (note that $Q_{n-1}^{n}\left(\bar{f}_{0}\right)=A_{0}^{\wedge n}$, so that this covers the leftmost map in (3), and that latching conditions for limit ordinals are trivial). This now follows by applying Lemma 4.11 to $i_{\beta}, f_{\beta}$ and Lemma 4.10 to $A_{0} \stackrel{\bar{f}_{\beta}}{\longrightarrow} A_{\beta} \stackrel{f_{\beta}}{\longrightarrow} A_{\beta+1}$ (note that $\bar{f}_{\beta}^{\square n}, n \geqslant 0$ is a $S \Sigma$-inj $\Sigma_{n}$-proj cofibration by the induction hypothesis), finishing the proof of the main claim.

For the extra claim, note that applying the main claim of the result to the map $* \rightarrow A$ yields that $A^{\wedge \bar{n}}, \bar{n} \geqslant 0$ is $S \sum$-inj $\Sigma_{\bar{n}}$-proj cofibrant (since $Q_{\bar{n}-1}^{\bar{n}}(* \rightarrow A)=*$ ). The additional conditions in Lemma 4.10 are hence satisfied and the strengthened conclusions now allow us to conclude the $\kappa$-cofibrancy for $0 \leqslant \bar{n}<n$ of the vertical $\kappa$-diagram map ( $\kappa$-th map excluded)

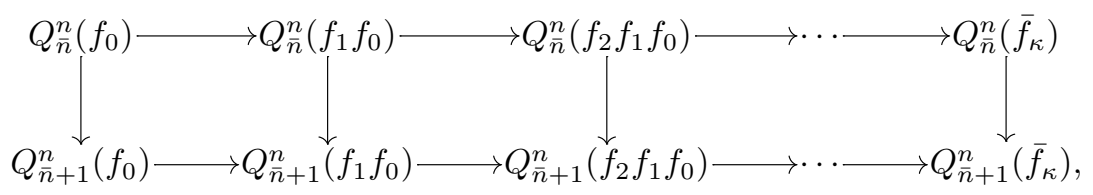

thereby showing $Q_{\bar{n}}^{n}\left(\bar{f}_{\kappa}\right) \rightarrow Q_{\bar{n}+1}^{n}\left(\bar{f}_{\kappa}\right)$ is a $S \Sigma$-inj $\Sigma_{n}$-proj cofibration. Since $Q_{0}^{n}\left(\bar{f}_{\kappa}\right)=$ $A^{\wedge n}$, this finishes the proof.

\section{Cofibrancy of operadic constructions}

The goal of this section is to prove Theorems 1.1, 1.4, 1.5, 1.6 and 1.8. 
Subsection 5.1 recalls some required operadic terminology and basic results.

Subsection 5.2 proves Proposition 5.20, a filtration result that is key to the proof of Theorem 1.1.

Subsection 5.3 extends the model structures of Section 3 to the category Sym of spectral symmetric sequences and proves for them analogues of the key results in Section 4.

Finally, the main proofs are found in Subsections 5.4 and 5.5.

\subsection{Definitions: operads, modules and algebras}

We now recall some standard operadic terminology. We do so in terms of a general closed symmetric monoidal category $\mathcal{C}$ in order to greatly streamline the proof of Theorem 1.1. Indeed, even when proving only the algebra case of Theorem 1.1, Definition 5.15 makes it necessary to nonetheless understand left modules, making it convenient to unify the discussion using Proposition 5.14.

Definition 5.1. Let $(\mathcal{C}, \otimes, \mathbb{1})$ denote a closed symmetric monoidal category.

The category $\operatorname{Sym}(\mathcal{C})$ of symmetric sequences in $\mathcal{C}$ is the category of functors $\Sigma \rightarrow \mathcal{C}$.

Further, for $G$ a finite group the category $\operatorname{Sym}^{G}(\mathcal{C})$ of $G$-symmetric sequences in $\mathcal{C}$ is the category of functors $G \rightarrow \operatorname{Sym}(\mathcal{C})$.

Remark 5.2. A symmetric sequence $X$ is formed by objects $X(r) \in \mathcal{C}, r \geqslant 0$ each with a left $\Sigma_{r}$-action. To avoid confusion when $\mathcal{C}=\mathrm{Sp}^{\Sigma}$ or a related category, we reserve the letter $r$ for this external index and keep $m$ as the internal spectrum index, so that $X_{m}(r)$ denotes the $m$-th simplicial set of $X(r)$.

We now recall the two usual monoidal structures on $\operatorname{Sym}(\mathcal{C})$. For our purposes the composition product $\circ$ is the most important of the two, with the tensor product $\check{\otimes}$ playing an auxiliary role.

Definition 5.3. Given $X, Y \in \operatorname{Sym}(\mathcal{C})$ we define their tensor product to be

$$
(X \check{\otimes} Y)(r)=\coprod_{0 \leqslant \bar{r} \leqslant r} \Sigma_{r} \sum_{\Sigma_{\bar{r}} \times \Sigma_{r-\bar{r}}} X(\bar{r}) \otimes Y(r-\bar{r})
$$

and their composition product to be

$$
(X \circ Y)(r)=\coprod_{\bar{r} \geqslant 0} X(\bar{r}) \otimes_{\Sigma_{\bar{r}}}\left(Y^{\check{\otimes} \bar{r}}(r)\right) .
$$

One has the following result (for a discussion of reflexive coequalizers see, for example, [3, Def. 3.26] and the propositions immediately following it).

Proposition 5.4. Let $(\mathcal{C}, \otimes, \mathbb{1})$ be a closed symmetric monoidal category with initial object $\varnothing$. Then

- (Sym, $\check{\otimes}, \check{1})$ is a closed symmetric monoidal category, with unit $\check{\mathbb{1}}(0)=\mathbb{1}, \check{\mathbb{1}}(r)=$ $\varnothing, r \geqslant 1$;

- (Sym,, $\mathcal{I})$ is a (non-symmetric) monoidal category, with unit $\mathcal{I}(1)=\mathbb{1}, \mathcal{I}(r)=$ $\varnothing, r \neq 1$.

Further, o commutes with all colimits in the first variable and with filtered colimits and reflexive coequalizers in the second variable. 
Definition 5.5. An operad $\mathcal{O}$ in $\mathcal{C}$ is a monoid object in $\operatorname{Sym}(\mathcal{C})$ with respect to $\circ$, i.e., a symmetric sequence $\mathcal{O}$ together with multiplication and unit maps

$$
\mathcal{O} \circ \mathcal{O} \rightarrow \mathcal{O}, \quad \mathcal{I} \rightarrow \mathcal{O}
$$

satisfying the usual associativity and unit conditions.

Definition 5.6. Let $\mathcal{O}$ be an operad in $\mathcal{C}$. A left module $N$ (resp., right module $M$ ) over $\mathcal{O}$ is an object in $\operatorname{Sym}(\mathcal{C})$ together with a map

$$
\mathcal{O} \circ N \rightarrow N \quad \text { (resp., } M \circ \mathcal{O} \rightarrow M)
$$

satisfying the usual associativity and unit conditions. The category of left modules (resp., right modules) over $\mathcal{O}$ is denoted $\operatorname{Mod}_{\mathcal{O}}^{l}\left(\right.$ resp., $\left.\operatorname{Mod}_{\mathcal{O}}^{r}\right)$. Further, left modules $X$ over $\mathcal{O}$ concentrated in degree 0 (i.e., such that $X(r)=\varnothing$ for $r \geqslant 1$ ) are called algebras over $\mathcal{O}$. The category of algebras over $\mathcal{O}$ is denoted $\mathrm{Alg}_{\mathcal{O}}$.

Proposition 5.7. The categories $\operatorname{Mod}_{\mathcal{O}}^{r}, \operatorname{Mod}_{\mathcal{O}}^{l}$ and $\operatorname{Alg}_{\mathcal{O}}$ have all small limits and colimits.

Further, all limits and colimits in $\operatorname{Mod}_{\mathcal{O}}^{r}$ are underlying in $\operatorname{Sym}(\mathcal{C})$, and likewise for all limits, filtered colimits and reflexive coequalizers in both $\operatorname{Mod}_{\mathcal{O}}^{l}$ and $\mathrm{Alg}_{\mathcal{O}}$.

Definition 5.8. Given $M \in \operatorname{Mod}_{\mathcal{O}}^{r}, N \in \operatorname{Mod}_{\mathcal{O}}^{l}$, their relative composition product is the reflexive coequalizer

$$
M \circ_{\mathcal{O}} N=\operatorname{colim}(M \circ \mathcal{O} \circ N \rightrightarrows M \circ N) .
$$

Lemma 5.9. Consider the bifunctors

$$
\operatorname{Mod}_{\mathcal{O}}^{l} \times \operatorname{Sym}(\mathcal{C}) \stackrel{-\circ-}{\longrightarrow} \operatorname{Mod}_{\mathcal{O}}^{l}, \quad \operatorname{Mod}_{\mathcal{O}}^{r} \times \operatorname{Mod}_{\mathcal{O}}^{l} \stackrel{-0^{O-}}{\longrightarrow} \operatorname{Sym}(\mathcal{C}) .
$$

o preserves any colimit in the $\operatorname{Mod}_{\mathcal{O}}^{l}$ variable and $\circ_{\mathcal{O}}$ preserves reflexive coequalizers and filtered colimits in the $\operatorname{Mod}_{\mathcal{O}}^{l}$ variable.

Proof. Since any $M \in \operatorname{Mod}_{\mathcal{O}}^{l}$ is a reflexive coequalizer $\operatorname{colim}(\mathcal{O} \circ \mathcal{O} \circ M \rightrightarrows \mathcal{O} \circ M)$ of free left modules, it suffices to verify the claim for diagrams of free left modules and free maps, and for those the result follows by Proposition 5.4.

Remark 5.10. We will also use the analogue of Definition 5.6 for the category $\operatorname{Sym}^{G}(\mathcal{C})$. One has a formal analogue of Proposition 5.4 for $\operatorname{Sym}^{G}(\mathcal{C})$ using the same monoidal structures $\ddot{\otimes}$ and $\circ$ (with diagonal $G$-action) and units (with trivial $G$-action), so that operads and their left modules, right modules and algebras in $\operatorname{Sym}^{G}(\mathcal{C})$ are defined just as above.

Iterating the Sym construction will allow us to use Proposition 5.14 to reduce the study of left modules to that of algebras.

Definition 5.11. The category $B \operatorname{Sym}(\mathcal{C})$ of bi-symmetric sequences in $\mathcal{C}$ is the category $\operatorname{Sym}(\operatorname{Sym}(\mathcal{C}))$ of symmetric sequences of symmetric sequences in $\mathcal{C}$. 
Remark 5.12. Since an object $X \in \operatorname{BSym}(\mathcal{C})$ is formed by objects $X(r, s) \in \mathcal{C}, r, s \geqslant 0$ with $\Sigma_{r} \times \Sigma_{s}$-actions one has two different inclusions

$$
(-)^{\mathrm{r}}: \operatorname{Sym}(\mathcal{C}) \hookrightarrow \operatorname{BSym}(\mathcal{C}), \quad(-)^{\mathrm{s}}: \operatorname{Sym}(\mathcal{C}) \hookrightarrow \operatorname{BSym}(\mathcal{C})
$$

defined by

$$
X^{\mathrm{r}}(r, s)=\left\{\begin{array}{ll}
X(r), & \text { if } s=0 \\
\varnothing, & \text { if } s \neq 0
\end{array}, \quad X^{\mathrm{s}}(r, s)=\left\{\begin{array}{ll}
X(s), & \text { if } r=0 \\
\varnothing, & \text { if } r \neq 0
\end{array} .\right.\right.
$$

Following Definition 5.3 one can build two monoidal structures in $\operatorname{BSym}(\mathcal{C})$ which we denote by $\check{\otimes}$ and $\check{o r}^{r}$. Here we mark the composition product ór to indicate that $r$ is kept as the operadic index. Note that while $\ddot{\otimes}$ behaves symmetrically with respect to the indexes $r$ and $s, \check{o r}$ does not.

Both of the following results follow by a straightforward calculation.

Proposition 5.13. $(-)^{r},(-)^{\mathrm{s}}$ are monoidal functors from the symmetric monoidal structure $\ddot{\otimes}$ to the symmetric monoidal structure $\check{\otimes}$.

$(-)^{r}$ is a monoidal functor from the monoidal structure o to the monoidal structure ór.

Proposition 5.14. Let $\mathcal{O}$ be an operad in $\mathcal{C}$. There is a natural isomorphism of categories

$$
(-)^{\mathrm{s}}: \operatorname{Mod}_{\mathcal{O}}^{l}(\mathcal{C}) \stackrel{\simeq}{\rightarrow} \operatorname{Alg}_{\mathcal{O} r}(\operatorname{Sym}(\mathcal{C}))
$$

\subsection{Filtrations}

This subsection proves Proposition 5.20, which provides the key filtrations to prove Theorem 1.1. These filtrations are adapted from $[\mathbf{1}, \mathbf{3}]$, among others, except we here show that such filtrations also hold after applying $M \circ_{\mathcal{O}}(-)$ for some $M \in \operatorname{Mod}_{\mathcal{O}}^{r}$. This is partly enabled by an alternate definition of $\mathcal{O}_{A}$.

Definition 5.15. Let $\mathcal{O}$ be an operad in $\mathcal{C}$ and $A \in A \lg _{\mathcal{O}}$ regarded as an element of $\operatorname{Mod}_{\mathcal{O}}^{l}$. We define

$$
\mathcal{O}_{A}=\mathcal{O} \amalg A,
$$

were the coproduct is taken in $\operatorname{Mod}_{\mathcal{O}}^{l}$. Additionally, for $M \in \operatorname{Mod}_{\mathcal{O}}^{r}$ we define

$$
M_{A}=M \circ_{\mathcal{O}} \mathcal{O}_{A} \text {. }
$$

Remark 5.16. As noted to the author by Harper, (5) appeared as [5, Prop. 5.52]. However, we benefit from using (5) as our definition of $\mathcal{O}_{A}$, hence streamlining the proofs of Propositions 5.18, 5.19 versus similar results in [5].

Remark 5.17. There are adjunctions

$$
\iota: \operatorname{Alg}_{\mathcal{O}} \rightleftarrows \operatorname{Mod}_{\mathcal{O}}^{l}:(-)(0) \quad(-)(0): \operatorname{Mod}_{\mathcal{O}}^{l} \rightleftarrows \operatorname{Alg}_{\mathcal{O}}: \widetilde{(-)},
$$

where $\iota$ is the inclusion and $\tilde{A}(0)=A, \tilde{A}(r)=\varnothing$ for $r \geqslant 1$. In particular, colimits in $\mathrm{Alg}_{\mathcal{O}}$ can be computed after the inclusion into $\operatorname{Mod}_{\mathcal{O}}^{l}$ and $\mathcal{O}_{A}(0)=A$. 
Proposition 5.18. Let $A \in \operatorname{Alg}_{\mathcal{O}}$ and $X \in \operatorname{Sym}(\mathcal{C})$. Then there is a natural isomorphism of $\operatorname{Mod}_{\mathcal{O}}^{l}$-valued functors

$$
(\mathcal{O} \circ X) \amalg A \simeq \mathcal{O}_{A} \circ X=\coprod_{r \geqslant 0} \mathcal{O}_{A}(r) \otimes_{\Sigma_{r}} X^{\check{\otimes} r} .
$$

Additionally, for $M \in \operatorname{Mod}_{\mathcal{O}}^{r}$ there is a natural isomorphism of $\operatorname{Sym}(\mathcal{C})$-valued functors

$$
M \circ_{\mathcal{O}}((\mathcal{O} \circ X) \amalg A) \simeq M_{A} \circ X=\coprod_{r \geqslant 0} M_{A}(r) \otimes_{\Sigma_{r}} X^{\check{\otimes} r} .
$$

Proof. We compute (applying Lemma 5.9 to the coproduct $\mathcal{O} \amalg A$ )

$$
\mathcal{O}_{A} \circ X=(\mathcal{O} \amalg A) \circ X \simeq(\mathcal{O} \circ X) \amalg(A \circ X)=(\mathcal{O} \circ X) \amalg A,
$$

where $A \circ X=A$ since $A$ is in degree 0 . The additional claim is obvious.

Proposition 5.19. Given $M \in \operatorname{Mod}_{\mathcal{O}}^{r}, X \in \mathcal{C}$ and $A \in \mathrm{Alg}_{\mathcal{O}}$ one has natural isomorphisms of $\operatorname{Sym}(\mathcal{C})$-valued functors

$$
M_{\mathcal{O} X \amalg A}(-)=\left(M \circ_{\mathcal{O}}(\mathcal{O} \amalg \mathcal{O} \circ X \amalg A)\right)(-) \simeq \coprod_{r \geqslant 0} M_{A}(r+(-)) \otimes_{\Sigma_{r}} X^{\otimes r} .
$$

Proof. This follows formally using the $(-)^{\mathrm{r}},(-)^{\mathrm{s}}$ functors. Combining Proposition 5.14 to change perspective to $\operatorname{Alg}_{\mathcal{O}^{r}}(\operatorname{Sym}(\mathcal{C}))$ with Proposition 5.18 yields

$$
\left(M \circ_{\mathcal{O}}(\mathcal{O} \amalg \mathcal{O} \circ X \amalg A)\right)^{s} \simeq M^{r} \circ_{\mathcal{O}^{r}}^{r}\left(\mathcal{O}^{s} \amalg \mathcal{O}^{r} \circ^{r} X^{s} \amalg A^{s}\right) \simeq M_{\mathcal{O}^{s} \amalg A^{s}}^{\mathrm{s}} \circ^{r} X^{s} .
$$

Applying Proposition 5.18 and noting $A^{\mathrm{s}}=A^{\mathrm{r}}$ (as $A$ is an algebra) we compute

$$
\begin{aligned}
M_{\mathcal{O}^{s} \amalg A^{s}}^{r} & =M^{r} \circ_{\mathcal{O}^{r}}^{r}\left(\mathcal{O}^{r} \amalg \mathcal{O}^{s} \amalg A^{s}\right)=M^{r} \circ_{\mathcal{O}^{r}}^{r}\left(\mathcal{O}^{r} \circ^{r}\left(\mathcal{I}^{r} \amalg \mathcal{I}^{s}\right) \amalg A^{s}\right) \simeq \\
& \simeq M_{A^{s}}^{r} \circ^{r}\left(\mathcal{I}^{r} \amalg \mathcal{I}^{s}\right)=M_{A^{r}}^{r} \circ^{r}\left(\mathcal{I}^{r} \amalg \mathcal{I}^{s}\right) \simeq\left(M_{A}\right)^{r} \circ^{r}\left(\mathcal{I}^{r} \amalg \mathcal{I}^{s}\right),
\end{aligned}
$$

showing $M_{\mathcal{O}^{s} \amalg A^{\mathrm{s}}}^{\mathrm{s}}(r, s) \simeq M_{A}(r+s)$. Plugging into (6) finishes the proof.

We now turn to the key result in the subsection. $Q_{r-1}^{r}(f)$ is defined in Remark 4.5.

Proposition 5.20. Consider any pushout in $\operatorname{Alg}_{\mathcal{O}}(\mathcal{C})$ of the form

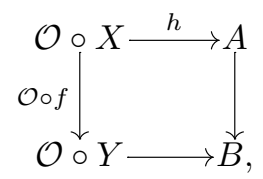

and let $M \in \operatorname{Mod}_{\mathcal{O}}^{r}$. Then, in the underlying category $\mathcal{C}$,

$$
M \circ_{\mathcal{O}} B \simeq \operatorname{colim}\left(A_{0}^{M} \rightarrow A_{1}^{M} \rightarrow A_{2}^{M} \rightarrow \cdots\right),
$$

where $A_{0}^{M}=M \circ_{\mathcal{O}} A$ and the $A_{r}^{M}$ are built inductively from pushout diagrams

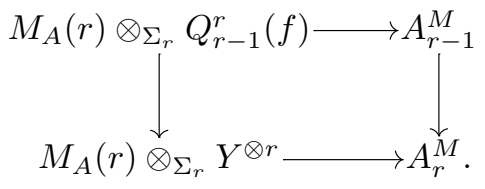


Remark 5.21. To streamline the proof of Theorem 1.1 we will need to apply Proposition 5.20 to the category $\operatorname{Mod}_{\mathcal{O}}^{l}$, a move enabled by Proposition 5.14. This is mostly straightforward, with occurrences of $\mathcal{C}$ replaced by $\operatorname{Sym}(\mathcal{C})$ and $\otimes$ replaced by $\check{\otimes}$, though defining $M_{N} \in \operatorname{BSym}(\mathcal{C})$ when $N \in \operatorname{Mod}_{\mathcal{O}}^{l}$ requires some care. Analyzing Proposition 5.14 and Definition 5.15 leads to the definition

$$
M_{N}=M_{N^{s}}^{\mathrm{r}}=M^{\mathrm{r}} \check{\check{o}}_{\mathcal{O}^{r}}^{\mathrm{r}}\left(\mathcal{O}^{\mathrm{r}} \amalg N^{\mathrm{s}}\right) \text {. }
$$

Note that this is compatible with Definition 5.15 when $N=A$ is an algebra since then $A^{\mathrm{s}}=A^{\mathrm{r}}$ so that $M_{A^{\mathrm{s}}}^{\mathrm{r}}=M_{A^{\mathrm{r}}}^{\mathrm{r}}=\left(M_{A}\right)^{\mathrm{r}}$.

Remark 5.22. When $M=\mathcal{O},(10)$ appeared as [5, Prop. 5.52], albeit with transposed indexes due to differing conventions. Our convention has one nice advantage: filtrations of $M_{A}$ follow by Remark 5.21 without a separate proof.

The remainder of the subsection is dedicated to proving Proposition 5.20. We essentially adapt the proof of [3, Prop. 4.20], although we substantially repackage the argument using a more categorical perspective.

For motivation we note that, in short, the proof of [3, Prop. 4.20] follows by noticing that $A \amalg_{\mathcal{O} \circ X} \mathcal{O} \circ Y$ is built out of terms of the form

$$
\mathcal{O}_{A}(|w|) \otimes w^{\otimes}(X, Y)
$$

where $w^{\otimes}(X, Y)$ denotes a word (or non-abelian monomial) for the operation $\otimes$ in the letters $X, Y$ (e.g., $X \otimes Y \otimes X, Y \otimes Y \otimes X \otimes Y$ ) and $|w|$ its length, glued along certain maps between them. ${ }^{3}$ The filtration (8) is then obtained by analyzing a long list of compatibility relations satisfied by those maps.

One drawback of such an approach is that it can be hard to keep track of the compatibilities that need to be verified. Instead, our approach will be to first identify a "diagram category of words" $\mathcal{W}$ together with a functor $F: \mathcal{W} \rightarrow \mathcal{C}$ defined on objects by (11) and for which $A \amalg_{\mathcal{O} \circ X} \mathcal{O} \circ Y=\operatorname{colim}_{\mathcal{W}} F$. Then, since $\mathcal{W}$ will encode all the necessary maps and compatibilities, the desired filtration (8) will follow from a filtration $\mathcal{W}_{\leqslant r}$ of $\mathcal{W}$ itself.

To motivate the definition, note that $\mathcal{W}$ needs enough arrows to describe: (i) the $Q_{r-1}^{r}$ constructions of Remark 4.5, i.e., $\mathcal{W}$ should contain the " $r$-cube categories" $(x \rightarrow y)^{\times r}$; (ii) the $\Sigma_{r}$-action on $Q_{r-1}^{r}$; (iii) maps between the terms in (11) that remove some $X$ letters (induced by $h: \mathcal{O} \circ X \rightarrow A$ in (7)).

This desiderata will likely remind some readers of Grothendieck constructions (cf., for example, [12, Construction 7.1.9]).

Definition 5.23. Consider the functor (Fininj denoting (a skeleton of) finite sets and injections) $(x \rightarrow y)^{(-)}$: Fininj $^{o p} \rightarrow$ Cat defined by $\underline{r}=\{1,2, \cdots, r\} \mapsto(x \rightarrow y)^{\underline{r}}, r \geqslant 0$ and let $\mathcal{G}$ denote the corresponding Grothendieck construction. Explicitly, objects of

\footnotetext{
${ }^{3}$ We note, however, that the need to deal with non-abelian monomials, rather than abelian ones, is somewhat hidden in the presentation of the proof in $[\mathbf{3}]$. We recommend the reader interested in gleaning why these are needed to focus on the last two paragraphs of that proof.
} 
$\mathcal{G}$ are pairs

$$
\left(\underline{r}, w \in(x \rightarrow y)^{\underline{r}}\right)
$$

and an arrow $(\underline{r}, w) \rightarrow\left(\underline{r_{*}}, w_{*}\right)$ is a pair

$$
\left(\iota: \underline{r_{*}} \hookrightarrow \underline{r}, w \circ \iota \rightarrow w_{*}\right)
$$

(note that, since $(x \rightarrow y) \stackrel{r_{*}}{\underline{n}}$ is a poset, arrows are determined by their first component) with composition defined in the obvious way.

Notation 5.24. To ease notation we will when convenient refer to an object of $\mathcal{G}$ by its second component $w$ and to an arrow by its first component $\iota$.

Remark 5.25. Objects $(\underline{r}, w) \in \mathcal{G}$ can be identified with words $w(x, y)$ on letters $x$ and $y$ where $r=|w|$, the length of the word. Further, we let $|w|_{x}$ (resp., $|w|_{y}$ ) denote the number of $x$ 's (resp., $y$ 's) in $w$.

Given a word $w=l_{1} l_{2} \cdots l_{r}$ with $l_{i} \in\{x, y\}$ and an injection $\iota: r_{*} \hookrightarrow \underline{r}$, one has $w \circ \iota=l_{\iota(1)} l_{\iota(2)} \cdots l_{\iota\left(r_{*}\right)}$, which we think of as the word obtained by removing the letters of $w$ in positions not in $\iota\left(r_{*}\right)$ and suitably shuffling the remaining letters. An arrow $w \rightarrow w_{*}$ can then be interpreted as an injection $\iota:\left|w_{*}\right| \hookrightarrow|w|$ such that, after removing and shuffling letters of $w$ to obtain $w \circ \iota$, one can obtain $w_{*}$ from $w \circ \iota$ by further replacing some $x$ 's with $y$ 's (now without shuffling).

Note that hence any $\iota: w \rightarrow w_{*}$ has a natural factorization $w \rightarrow w \circ \iota \rightarrow w_{*}$.

Comparing the description in Remark 5.25 with the desiderata for $\mathcal{W}$, we see that $\mathcal{G}$ has more arrows than desired, namely those that remove $y$ letters.

Definition 5.26. $\mathcal{W}$ is the subcategory of $\mathcal{G}$ with all objects and the arrows $\iota:(\underline{r}, w) \rightarrow\left(\underline{r_{*}}, w_{*}\right)$ such that $w\left(\underline{r}-\iota\left(\underline{r_{*}}\right)\right) \subset\{x\}$ or, equivalently, $|w|_{y}=|w \circ \iota|_{y}$.

Further, for each $r \geqslant 0$, let $\mathcal{W}_{\leqslant r}$ (resp., $\mathcal{W}_{r}$ ) denote the full subcategory of those $w \in \mathcal{W}$ satisfying $|w| \leqslant r$ (resp., $|w|=r$ ).

Notation 5.27. It will be convenient to name certain types of arrows in $\mathcal{W}$ :

- a shuffle is an arrow $\sigma: w \rightarrow w \circ \sigma$ for $\sigma \in \Sigma_{|w|}^{o p}$;

- a tidy arrow is an arrow $\pi: w=\bar{w} x^{a} \rightarrow w_{*}$ for $\pi$ the inclusion $\underline{\left|w_{*}\right|}=\underline{|\bar{w}|} \subset \underline{|w|}$;

- a removing arrow is an arrow $w \rightarrow y^{|w|_{y}}$;

- a replacing arrow is an arrow $w \rightarrow y^{|w|}$.

Definition 5.28. $\mathcal{W}$ is the subcategory of $\mathcal{W}$ with the same objects but only the shuffles, removing and replacing arrows.

Remark 5.29. Keeping the intuition of Remark 5.25, tidy arrows remove some $x$ 's at the end of a word and then replace some $x$ 's by $y$ 's without any shuffling, removing arrows remove all $x$ 's (perhaps shuffling) and replacing arrows replace all $x$ 's by $y$ 's (perhaps shuffling).

The key to proving Proposition 5.20 are the following lemmas building $F: \mathcal{W} \rightarrow \mathcal{C}$ and establishing some categorical results about $\mathcal{W}$. 
Lemma 5.30. The pushout diagram (7) and $M \in \operatorname{Mod}_{\mathcal{O}}^{r}(\mathcal{C})$ naturally induce a functor

$$
F^{M}: \mathcal{W} \rightarrow \mathcal{C}
$$

Proof. We define $F^{M}$ on objects in the obvious way as

$$
F^{M}(w(x, y))=M_{A}(|w|) \otimes w^{\otimes}(X, Y) .
$$

For arrows, we first declare that for a shuffle $\sigma: w \rightarrow w \circ \sigma$,

$$
M_{A}(|w|) \otimes w^{\otimes}(X, Y) \stackrel{F^{M}(\sigma)}{\longrightarrow} M_{A}(|w|) \otimes(w \circ \sigma)^{\otimes}(X, Y)
$$

is the map defined by the action of $\sigma^{-1} \in \Sigma_{|w|}$ on $M_{A}(|w|)$ and by shuffling $w^{\otimes}(X, Y)$. Since any arrow can be made tidy by pre-composing with a shuffle, it remains to coherently define $F^{M}$ on tidy arrows. For a tidy arrow $\pi: w=\bar{w}(x, y) x^{a} \rightarrow w_{*}(x, y)$, define $F^{M}(\pi)$ via the diagram (with vertical maps the summand inclusions induced by Proposition 5.18 and writing $\mathcal{O}(-)$ for $\mathcal{O} \circ(-))$

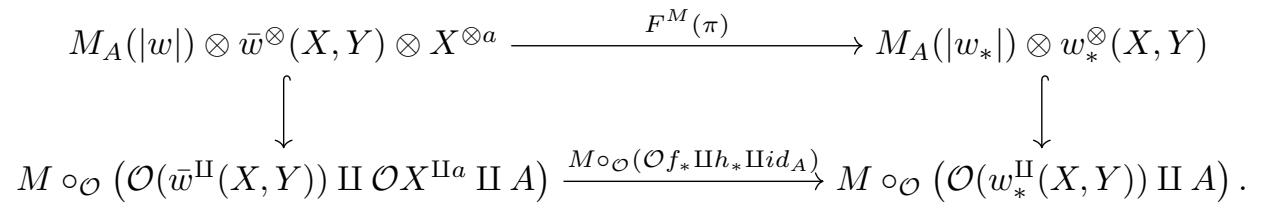

$F^{M}(\pi)$ is well defined since $\pi \sigma$ is tidy only for $\sigma \in \Sigma_{a} \subset \Sigma_{|w|}$ and such shuffles do not change (13). It follows that $F^{M}$ is well defined on all arrows.

We now verify $F^{M}$ respects compositions. This is clear when composing either two shuffles or two tidy arrows, and since general two-fold compositions factor as $w \stackrel{\sigma}{\rightarrow} w \circ \sigma \stackrel{\pi}{\rightarrow} w_{*} \stackrel{\sigma_{*}}{\rightarrow} w_{*} \circ \sigma_{*} \stackrel{\pi_{*}}{\rightarrow} w_{* *}$ with $\sigma, \sigma_{*}$ shuffles and $\pi, \pi_{*}$ tidy, it remains to show $F^{M}\left(\sigma_{*} \pi\right)=F^{M}\left(\sigma_{*}\right) F^{M}(\pi)$. Identifying $\sigma_{*} \in \Sigma_{\left|w_{*}\right|} \subset \Sigma_{|w|}$, one has $\sigma_{*} \pi \sigma_{*}^{-1}$ tidy, so that by definition $F^{M}\left(\sigma_{*} \pi\right)=F^{M}\left(\sigma_{*} \pi \sigma_{*}^{-1}\right) F^{M}\left(\sigma_{*}\right)$. The claim now follows since (13) respects the action of $\sigma_{*}$.

Recall that a functor $\mathcal{J} \rightarrow \mathcal{I}$ between diagram categories is called final if for any functor $F: \mathcal{I} \rightarrow \mathcal{C}$ (where $\mathcal{C}$ is a category with all small colimits) one has

$$
\operatorname{colim}_{\mathcal{I}} F=\left.\operatorname{colim}_{\mathcal{J}} F\right|_{\mathcal{J}}
$$

We will need several finality conditions for subcategories of $\mathcal{W}$. In all cases we show them by verifying (cf. [10, IX.3 Thm. 1]) that for all $i \in \mathcal{I}$ the under categories $i \downarrow \mathcal{J}$ are non-empty (this will always be obvious) and connected.

Lemma 5.31. The subcategory $\overline{\mathcal{W}}$ is final in $\mathcal{W}$.

Proof. $w \downarrow \overline{\mathcal{W}}$ is connected iff any two arrows in $\mathcal{W}$ with source $w$ are connected by a zigzag of post-compositions with arrows in $\overline{\mathcal{W}}$. For such an arrow $\iota: w \rightarrow w_{*}$ the natural decomposition $w \rightarrow w \circ \iota \rightarrow w_{*}$ satisfies $|w|_{y}=|w \circ \iota|_{y},|w \circ \iota|=\left|w_{*}\right|$, so that 
by picking any arrows $w_{*} \rightarrow y^{\left|w_{*}\right|}$ and $w \circ \iota \rightarrow y^{|w|_{y}}$ one has a diagram

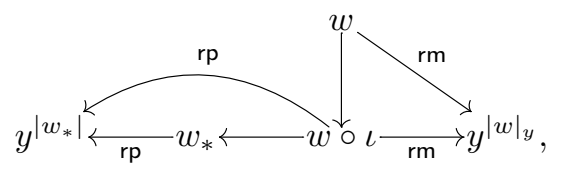

where arrows marked $\mathrm{rp}$ are replacing and arrows marked $\mathrm{rm}$ are removing. The marked arrows exhibit a zigzag in $w \downarrow \overline{\mathcal{W}}$ between $\iota$ and $i d_{w}$.

Lemma 5.32. Let $\overline{\mathcal{W}}_{y^{r}}$ denote the full subcategory of $\overline{\mathcal{W}}$ of objects that admit arrows to $y^{r}$. The group $\Sigma_{y^{r}}$ of shuffles of $y^{r}$ is final in $\overline{\mathcal{W}}_{y^{r}}$.

Proof. Since any $w$ is isomorphic up to shuffle to some $y^{b} x^{a}$, it suffices to check all $y^{b} x^{a} \downarrow \Sigma_{y^{r}}$ are connected, i.e., that all arrows $y^{b} x^{a} \rightarrow y^{r}$ in $\overline{\mathcal{W}}$ are connected by post-composing with a shuffle. Both cases $b=r$ and $a+b=r$ are clear.

Lemma 5.33. The subcategory $\mathcal{W}_{\leqslant(r-1)}$ is final in $\mathcal{W}_{\leqslant r}-y^{r}$.

Proof. When $|w| \leqslant r-1$ one has an initial object $i d_{w}$ in $w \downarrow \mathcal{W}_{\leqslant(r-1)}$, which is hence connected. When $|w|=r, w \downarrow \mathcal{W}_{\leqslant(r-1)}$ is connected precisely if any two arrows $w \rightarrow$ $w_{*}$ with $\left|w_{*}\right| \leqslant r-1$ are connected by a zigzag of post-compositions with arrows in $\overline{\mathcal{W}}_{\leqslant(r-1)}$. For any such arrow $\iota: w \rightarrow w_{*}$ the natural decomposition $w \rightarrow w \circ \iota \rightarrow w_{*}$ satisfies $|w|_{y}=|w \circ \iota|_{y} \leqslant r-1,|w \circ \iota|=\left|w_{*}\right| \leqslant r-1$ so that diagram (14) exhibits a zigzag between $\iota$ and an arrow $w \rightarrow y^{|w|_{y}}$. As in Lemma 5.32, all of the latter arrows are connected by post-composing with a shuffle (in fact, it suffices to check this for $\left.w=y^{b} x^{a}\right)$.

Lemma 5.34. $\mathcal{W}_{\leqslant r}=\left(\mathcal{W}_{\leqslant r}-y^{r}\right) \cup \mathcal{W}_{r}$. In fact, $N\left(\mathcal{W}_{\leqslant r}\right)=N\left(\mathcal{W}_{\leqslant r}-y^{r}\right) \cup N\left(\mathcal{W}_{r}\right)$.

Proof. Arrows in $\mathcal{W}$ never decrease $|-|_{y}$, hence any string of arrows in $\mathcal{W}_{\leqslant r}$ involving the object $y^{r}$ must in fact be a string of arrows in $\mathcal{W}_{r}$.

\section{Lemma 5.35.}

$$
M \circ_{\mathcal{O}} B \simeq \operatorname{colim}_{\mathcal{W}} F^{M} .
$$

Proof. Note first that by Lemma 5.31 it suffices to show $M \circ_{\mathcal{O}} B \simeq \operatorname{colim}_{\overline{\mathcal{W}}} F^{M}$.

By general considerations one can describe $B$ as a reflexive coequalizer

$$
B \simeq \operatorname{colim}((\mathcal{O} \circ(X \amalg Y) \amalg A) \rightrightarrows(\mathcal{O} \circ Y) \amalg A)
$$

and hence by Proposition 5.18 and Lemma 5.9

$$
M \circ_{\mathcal{O}} B \simeq \operatorname{colim}\left(M_{A} \circ(X \amalg Y) \underset{h_{*}}{\stackrel{f_{*}}{\longrightarrow}} M_{A} \circ Y\right) .
$$

Now note that

$$
M_{A} \circ(X \amalg Y)=\coprod_{i, j \geqslant 0} M_{A}(i+j) \otimes_{\Sigma_{i} \times \Sigma_{j}} X^{\otimes i} \otimes Y^{\otimes j},
$$

with the reflexive map in (15) naturally identifying $M_{A} \circ Y$ with the subobject formed by the $i=0$ summands. Since by naturality of Propositions 5.18 and 5.19 the maps 
being equalized in (15) send summands to summands, repackaging universal properties allows one to rewrite

$$
M \circ_{\mathcal{O}} B=\operatorname{colim}_{\mathcal{M}} \bar{F}^{M} .
$$

Here $\mathcal{M}$ is the diagram category whose objects we denote by monomials $x^{i} y^{j}, i, j \geqslant 0$ together with unique non-identity arrows $x^{i} y^{j} \rightarrow y^{i+j}, x^{i} y^{j} \rightarrow y^{j}$ for $i \neq 0$ (note that non-identity arrows can never be composed). $\bar{F}^{M}$ is defined on objects by

$$
\bar{F}^{M}\left(x^{i} y^{j}\right)=M_{A}(i+j) \otimes_{\Sigma_{i} \times \Sigma_{j}} X^{\otimes i} \otimes Y^{\otimes j}
$$

is induced on arrows $x^{i} y^{j} \rightarrow y^{i+j}$ by the map $f_{*}$ in (15) and on arrows $x^{i} y^{j} \rightarrow y^{j}$ by the map $h_{*}$.

There is an obvious functor $\overline{\mathcal{W}} \rightarrow \mathcal{M}$ defined by $w \mapsto x^{|w|_{x}} y^{|w|_{y}}$ (arrows are mapped in the only possible way and functoriality is trivial since non-identity arrows in $\mathcal{M}$ cannot be composed). We claim $\bar{F}^{M}=\operatorname{Lan}_{\overline{\mathcal{W}} \rightarrow \mathcal{M}} F^{M}$. By $[\mathbf{1 0}$, X.3.1]

$$
\left(\operatorname{Lan}_{\overline{\mathcal{W}} \rightarrow \mathcal{M}} F^{M}\right)\left(x^{i} y^{j}\right)=\left.\operatorname{colim}_{\overline{\mathcal{W}} \downarrow x^{i} y^{j}} F^{M}\right|_{\overline{\mathcal{W}} \downarrow x^{i} y^{j}} .
$$

When $i \neq 0, \overline{\mathcal{W}} \downarrow x^{i} y^{j}$ is just the groupoid of words $w$ with $|w|_{x}=i,|w|_{y}=j$, while for $y^{r}$ it is the category $\overline{\mathcal{W}}_{y^{r}}$ of Lemma 5.32 containing the final group $\Sigma_{y^{r}}$. In either case, the formula (16) computes the quotient of the terms in (12) by the obvious shuffle groupoid action and hence coincides with $\bar{F}^{M}$ on objects. To see (16) also coincides with $\bar{F}^{M}$ on arrows consider the commutative diagrams (with vertical maps induced by codiagonals and writing $\mathcal{O}(-)$ for $\mathcal{O} \circ(-))$
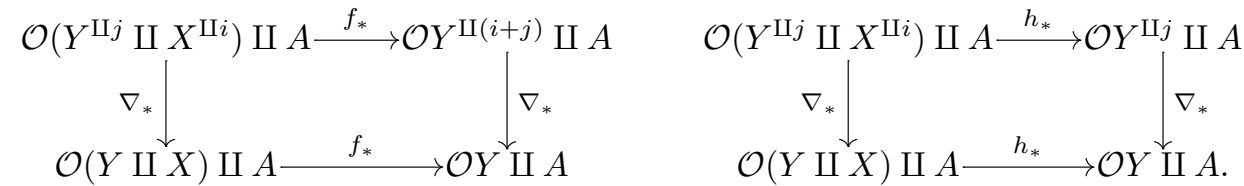

Since $F^{M}$ is defined using (shuffles) of the top maps, and $\bar{F}^{M}$ is defined using the bottom maps, we conclude (16) indeed equals $\bar{F}^{M}$ on maps. Noting that left Kan extensions have the same colimit finishes the proof.

Proof of Proposition 5.20. By the previous lemma $M \circ_{\mathcal{O}} B \simeq \operatorname{colim}_{\mathcal{W}} F^{M}$. We define $A_{r}^{M}=\operatorname{colim}_{\mathcal{W}_{\leqslant r}} F^{M}$, so that (8) is immediate since the $\mathcal{W}_{\leqslant r}$ filter $\mathcal{W}$. It is straightforward to check that Lemma 5.34 implies one has pushout diagrams

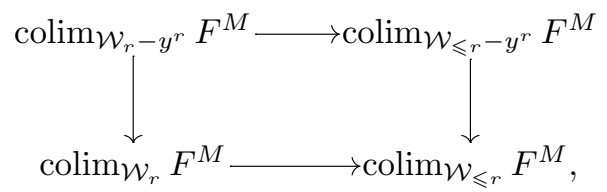

and it hence suffices to verify these diagrams have the form (9). The two diagrams coincide on the bottom right corner by definition and on the top right corner by Lemma 5.33. The left hand maps of the two diagrams are seen to coincide by direct computation since the tidy arrow subcategory of $\mathcal{W}_{r}$ is precisely $(x \rightarrow y)^{\underline{r}}$ and it is easy to check that $\operatorname{colim}_{\mathcal{W}_{r}} F^{M}=\left(\operatorname{colim}_{(x \rightarrow y)^{\underline{r}}} F^{M}\right)_{\Sigma_{r}}$ and similarly for $\mathcal{W}_{r}-y^{r}$.

\subsection{Model structures on Sym and Sym ${ }^{G}$}

Notation 5.36. In what follows we abbreviate $\operatorname{Sym}\left(\mathrm{Sp}^{\Sigma}\right)$ simply as Sym. 
We now introduce for Sym the analogues of the model structures in Section 3 and show that the main results in Section 4 formally imply their Sym analogues.

Definition 5.37. The $S$ stable (resp., monomorphism stable) model structure on Sym is obtained by combining the $S$ stable (resp., monomorphism stable) model structures on $\left(\mathrm{Sp}^{\Sigma}\right)^{\Sigma_{r}}$ in all degrees (cf. Section 3.3).

Definition 5.38. The positive $S$ stable model structure on Sym is the model structure obtained by combining the positive $S$ stable model structure in $\mathrm{Sp}^{\Sigma}$ on degree $r=0$ with the $S$ stable model structures on $\left(\mathrm{Sp}^{\Sigma}\right)^{\Sigma_{r}}$ in degrees $r \geqslant 1$ (cf. Sections 2.3 and 3.3).

Remark 5.39. To motivate the use of the word "positive" in the previous definition, recall that each $X \in$ Sym is composed of pointed simplicial sets $X_{m}(r)$, making it a bi-graded object. Since $\ddot{\otimes}$ is additive in both gradings, one can think of $m+r$ as the total degree of $X_{m}(r)$.

We will also want to have an analogue for $\operatorname{Sym}^{G}$ of the $\Sigma$-inj $G$-proj $S$ stable model structure on $\left(\mathrm{Sp}^{\Sigma}\right)^{G}$.

Definition 5.40. The $S \Sigma \times \Sigma$-inj G-proj stable model structure on $\mathrm{Sym}^{G}$ is the model structure obtained by combining the $S \Sigma \times \Sigma_{r}$-inj $G$-proj stable model structures on all degrees $r \geqslant 0$ (cf. Remark 3.14).

What follows are formal analogues for Sym of Propositions 4.1 and 4.2 and Theorems 1.3 and 1.2 .

Proposition 5.41. Suppose all categories are equipped with their respective $S \Sigma \times \Sigma$ inj G-proj stable model structure. Then the bifunctor

$$
\mathrm{Sym}^{G} \times \operatorname{Sym}^{\bar{G}} \stackrel{-\bar{\lambda}-}{\longrightarrow} \mathrm{Sym}^{G \times \bar{G}}
$$

is a left Quillen bifunctor.

Proof. Existence of the right adjoints is formal. Now recall that

$$
(X \check{\otimes} Y)(r)=\bigvee_{0 \leqslant \bar{r} \leqslant r} \Sigma_{r} \dot{\Sigma}_{\bar{r} \times \dot{\Sigma}_{r-\bar{r}}} X(\bar{r}) \wedge Y(r-\bar{r}) .
$$

By injectiveness of the model structures (cf. Remark 3.14), we can ignore the symmetric group actions, so that $\check{\wedge}$ is a wedge of bifunctors for each of which Proposition 4.1 applies.

Proposition 5.42. Let $\bar{G} \subset G$ be finite groups, and suppose each category is equipped with the respective $S \Sigma \times \Sigma$-inj G-proj stable model structure. Then both adjunctions

$$
\text { fgt: Sym }{ }^{G} \rightleftarrows \operatorname{Sym}^{\bar{G}}:\left((-)^{G \cdot S}\right)^{\bar{G}} \quad \text { and } \quad G \times{ }_{\bar{G}}(-):\left(\operatorname{Sym}^{\Sigma}\right)^{\bar{G}} \rightleftarrows\left(\operatorname{Sym}^{\Sigma}\right)^{G}: \mathrm{fgt}
$$

are Quillen adjunctions.

Proof. This is obvious from Proposition 4.2 since we are dealing with injective model structures (cf. Remark 3.14). 
Proposition 5.43. Consider the bifunctor

$$
\mathrm{Sym}^{G} \times \mathrm{Sym}^{G} \stackrel{-\check{\Lambda}_{G}-}{\longrightarrow} \mathrm{Sym},
$$

where the first copy of $\mathrm{Sym}^{G}$ is regarded as equipped with the $S \Sigma \times \Sigma$-inj G-proj stable model structure. Then $\check{\wedge}_{G}$ is a left Quillen bifunctor if either:

(a) Both the second $\mathrm{Sym}^{G}$ and the target Sym are equipped with the respective monomorphism stable model structures;

(b) Both the second $\mathrm{Sym}^{G}$ and the target Sym are equipped with the respective $S$ stable model structures.

Proof. This follows immediately by combining the "wedge of bifunctors" argument from the proof of Proposition 5.41 with Theorem 1.3.

Proposition 5.44. Let Sym be equipped with the positive $S$ stable model structure and $\mathrm{Sym}^{\Sigma_{n}}$ with the $S \Sigma \times \Sigma$-inj $\Sigma_{n}$-proj stable model structure.

Then for $f: A \rightarrow B$ a cofibration in Sym its $n$-fold pushout product

$$
f^{\square n}: Q_{n-1}^{n}(f) \rightarrow B^{n}
$$

is a cofibration in $\operatorname{Sym}^{\Sigma_{n}}$, which is a weak equivalence when $f$ is.

Furthermore, if $A$ is cofibrant in Sym then $Q_{n-1}^{n}(f)$ (resp., $\left.f^{\check{\wedge} n}: A^{\check{\wedge} n} \rightarrow B^{\check{\wedge} n}\right)$ is cofibrant (resp., cofibration between cofibrant objects) in Sym $^{\Sigma_{n}}$.

Proof. Note first that by injectiveness (cf. Remark 3.14) we need only worry about the $\Sigma_{n}$-actions and can ignore the $\Sigma_{r}$-actions.

Computing $X_{1} \check{\wedge} \cdots \check{\wedge} X_{n}$ iteratively and regrouping terms we get

$$
\left(X_{1} \check{\wedge} \check{\wedge} X_{n}\right)(r)=\bigvee_{\{\phi: \underline{r} \rightarrow \underline{n}\}} X_{1}\left(\phi^{-1}(1)\right) \wedge \cdots \wedge X_{n}\left(\phi^{-1}(n)\right) .
$$

Since the shuffle isomorphisms for $\check{\wedge}$ involve a post-composition $\Sigma_{n}$-action on the set $\{\phi: \underline{r} \rightarrow \underline{n}\}$ indexing the wedge summands, the $\Sigma_{n}$-coset decomposition

$$
\left(X_{1} \check{\wedge} \cdots \check{\wedge} X_{n}\right)(r)=\bigvee_{(\bar{\phi}) \in\{\phi: \underline{r} \rightarrow \underline{n}\} / \Sigma_{n}} \bigvee_{\phi \in(\bar{\phi})} X_{1}\left(\phi^{-1}(1)\right) \wedge \cdots \wedge X_{n}\left(\phi^{-1}(n)\right)
$$

is compatible with those shuffle isomorphisms, so that it suffices to verify the conclusions of the theorem for each of the subfunctors formed by the wedge summands over a single coset $(\bar{\phi}) \in\{\phi: \underline{r} \rightarrow \underline{n}\} / \Sigma_{n}$.

Now consider a map $f: A \rightarrow B$ in Sym. Without loss of generality we can assume that the representative $\bar{\phi}$ misses precisely the first $\bar{n}$ elements in $n$, so that when computing $f^{\square n}$ the $\Sigma_{n}$-isotropy of the $\bar{\phi}$ wedge summand (i.e., the subgroup sending that summand to itself) is $\Sigma_{\bar{n}}$, and hence the component of $f^{\square n}$ corresponding to the $(\bar{\phi})$ subfunctor in (17) can be rewritten as

$$
\Sigma_{n} \underset{\Sigma_{\bar{n}}}{\cdot} f(0)^{\square^{\wedge} \bar{n}} \square^{\wedge} f\left(\phi^{-1}(\bar{n}+1)\right) \square^{\wedge} \cdots \square^{\wedge} f\left(\phi^{-1}(n)\right) .
$$

We need to show that this is a $S \Sigma$-inj $\Sigma_{n}$-proj cofibration if $f$ is a positive $S$ cofibration. This follows by first applying Theorem 1.2 to $f(0)^{\square^{\wedge}} \bar{n}$, then applying 
Proposition 4.1 to conclude $f(0)^{\square^{\wedge} \bar{n}} \square^{\wedge} f\left(\phi^{-1}(\bar{n}+1)\right) \square^{\wedge} \ldots \square^{\wedge} f\left(\phi^{-1}(n)\right)$ is a $S$ $\Sigma$-inj $\Sigma_{\bar{n}}$-proj cofibration, and finishing by applying Proposition 4.2.

The additional claims assuming $A$ is positive $S$ cofibrant follow by the same argument by noting that $f_{1} \square^{\wedge} f_{2}, f_{1} \wedge f_{2}$ are cofibrations between cofibrant objects if so are $f_{1}, f_{2}$ and using the additional statements in Theorem 1.2.

Remark 5.45. All definitions and results in this subsection generalize to the category BSym $=\operatorname{Sym}($ Sym $)$. Indeed, one can define monomorphism, $S$ and positive $S$ stable model structures on BSym and $S \Sigma \times \Sigma \times \Sigma$-inj $\Sigma_{n}$-proj stable model structures on BSym $^{\Sigma_{n}}$ by just repeating Definitions 5.37, 5.38 and 5.40 except now replacing the initial structures on $\mathrm{Sp}^{\Sigma}$ with their eponymous analogues on Sym. Further, analyzing the proofs of Propositions 5.41, 5.42, 5.43 and 5.44 it is clear that those results themselves imply the analogue BSym results.

\subsection{Proof of Theorem 1.1}

Proof of Theorem 1.1. To simplify the discussion and notation somewhat, we first deal with the case where $f_{2}$ is a map in $\operatorname{Alg}_{\mathcal{O}} \subset \operatorname{Mod}_{\mathcal{O}}^{l}$.

Writing $f_{2}: A \rightarrow B$, note first that if $A=\mathcal{O}(0)$, then $f_{1} \circ_{\mathcal{O}} A$ is a $S$ cofibration (resp., monomorphism), since $f_{1} \circ_{\mathcal{O}} \mathcal{O}(0) \simeq f_{1} \circ_{\mathcal{O}} \mathcal{O} \circ * \simeq f_{1} \circ * \simeq f_{1}(0)$. Otherwise, the same conclusion follows by first running the full proof for the map $\mathcal{O}(0) \rightarrow A$.

We now write $f_{2}$ as a retract of a transfinite composition of a $\kappa$-diagram $\mathrm{F}: \kappa \rightarrow$ $\mathrm{Sp}^{\Sigma}$ where each successor map is a pushout of a generating cofibration, just as in (2). As usual, retracts cause no difficulty so we reduce to the case of $f_{2}$ the transfinite composition of $\mathrm{F}$. Recalling that $\circ_{\mathcal{O}}$ commutes with transfinite compositions in the second variable (cf. Lemma 5.9) and setting $f_{1}: M \rightarrow N$, one sees that $f_{1} \square^{\circ} \mathcal{O} f_{2}$ will be a suitable cofibration provided that $M \circ_{\mathcal{O}} \mathrm{F} \rightarrow N \circ_{\mathcal{O}} \mathrm{F}$ is a $\kappa$-projective cofibration between $\kappa$-diagrams. Since cofibrancy at $\beta=0$ is satisfied due to the previous paragraph, this amounts to verifying the cofibrancy of $f_{1} \square^{\circ} \mathcal{O} \mathrm{F}(\beta \rightarrow \beta+1)$ for $\beta<\kappa$ (the condition for limit ordinals being automatic since $\circ_{\mathcal{O}}$ commutes with transfinite compositions in the second variable). One hence reduces to the case where $f_{2}: A \rightarrow B$ is the pushout of a generating cofibration $\mathcal{O} \circ X \rightarrow \mathcal{O} \circ Y$, such as in Proposition 5.20. Borrowing the notation from that proposition we see that it suffices to show that the vertical map of filtration $\omega$-diagrams (recall that $\omega$ denotes $(0 \rightarrow 1 \rightarrow 2 \rightarrow \cdots))$

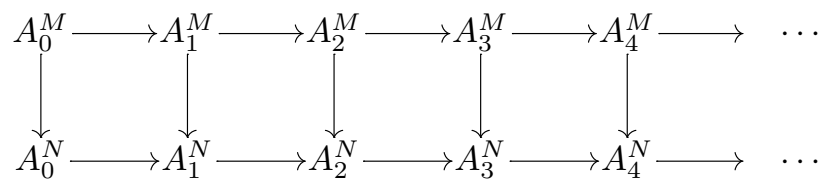

is a suitable $\omega$-projective cofibration. More explicitly, we need to show that each of the "pushout corner maps" $A_{r}^{M} \bigsqcup_{A_{r-1}^{M}} A_{r-1}^{N} \rightarrow A_{r}^{N}, r \geqslant 0$ (note that $A_{-1}^{M}=A_{-1}^{N}=\times$ *) is a $S$ cofibration (resp., monomorphism). Using the inductive description (9) this reduces to showing that the "pushout corner maps" of the diagrams

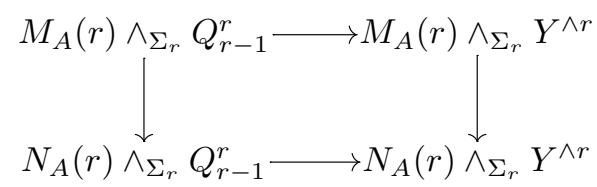


are themselves $S$ cofibrations (resp., monomorphisms). Combining Theorems 1.2 and 1.3 this reduces to showing that $M_{A}(r) \rightarrow N_{A}(r), r \geqslant 0$ is a $S$ cofibration (resp., monomorphism), or rather, that $M_{A} \rightarrow N_{A}$ is a $S$ cofibration (resp., monomorphism) in Sym. Recalling from Definition 5.15 that $M_{A} \rightarrow N_{A}$ can be written as

$$
M \circ_{\mathcal{O}}(\mathcal{O} \amalg A) \rightarrow N \circ_{\mathcal{O}}(\mathcal{O} \amalg A),
$$

we see that this last claim would follow directly from a different instance of the theorem we are trying to prove, namely the case of the maps $f_{1}: M \rightarrow N$ in $\operatorname{Mod}_{\mathcal{O}}^{r}$ and $\tilde{f}_{2}: \mathcal{O} \rightarrow \mathcal{O} \amalg A$ in $\operatorname{Mod}_{\mathcal{O}}^{l}$. Since $A$ is assumed cofibrant, it can be written as a retract of a transfinite composition of pushouts of generating cofibrations, and one hence reduces to the case $A=\operatorname{colim}_{\beta<\kappa} A_{\beta}$ where each $A_{\beta} \rightarrow A_{\beta+1}$ is the pushout of some generating positive $S$ cofibration $\mathcal{O} X_{\beta} \rightarrow \mathcal{O} Y_{\beta}$ in $\mathrm{Alg}_{\mathcal{O}}$.

Note now that one can repeat all of the arguments so far for $f_{1}$ and for the filtration $\tilde{f}_{2, \beta}: \mathcal{O} \amalg A_{\beta} \rightarrow \mathcal{O} \amalg A_{\beta+1}$ of the map $\tilde{f}_{2}: \mathcal{O} \rightarrow \mathcal{O} \amalg A$. Firstly, repeating the " $\kappa$-projective cofibration" argument, the $\beta=0$ condition is now that $f_{1} \circ_{\mathcal{O}} \mathcal{O}=f_{1}$ is a $S$ cofibration (resp., monomorphism), which is just one of the hypotheses, and the limit ordinal condition is again automatic. One hence reduces to showing, by induction on $\beta<\kappa$, that the theorem holds for $f_{1}$ and each $\tilde{f}_{2, \beta}$. Since $\tilde{f}_{2, \beta}$ is a pushout of $\mathcal{O} X_{\beta} \rightarrow \mathcal{O} Y_{\beta}$, one again reduces to showing that the map of filtration diagrams (built using Proposition 5.20 as described in Remark 5.21)

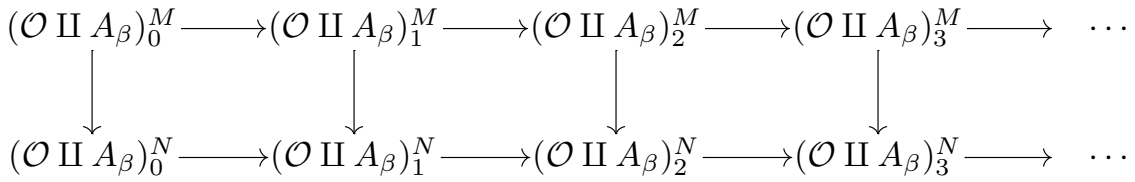

is a suitable $\omega$-cofibration, and again one reduces to checking that the pushout corner maps of each diagram

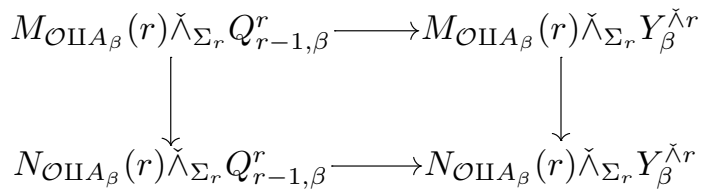

are $S$ cofibrations (resp., monomorphisms) in Sym. Arguing as before (but replacing uses of Theorems 1.2 and 1.3 by uses of their Sym analogues Propositions 5.44 and 5.43) one reduces to checking that $M_{\mathcal{O} \amalg A_{\beta}} \rightarrow N_{\mathcal{O} \amalg A_{\beta}}$ is a $S$ cofibration (resp., monomorphism) in BSym. The result now follows from the calculation in the proof of Proposition 5.19, which identifies $M_{\mathcal{O} \amalg A_{\beta}}(r, s) \rightarrow N_{\mathcal{O} \amalg A_{\beta}}(r, s)$ with $M_{A_{\beta}}(r+s) \rightarrow$ $N_{A_{\beta}}(r+s)$, together with the transfinite induction hypothesis (which, explicitly, states that $\left(M_{A_{\gamma}} \rightarrow N_{A_{\gamma}}\right)_{\gamma \leqslant \beta}$ is a projective cofibration).

Tracing through the steps above we also see that indeed $f_{1} \square^{\circ} \mathcal{O} f_{2}$ will be a weak equivalence if either $f_{1}$ or the original $f_{2}$ is.

Finally, we explain what changes when $f_{2}$ is a general cofibration between cofibrant objects in $\operatorname{Mod}_{\mathcal{O}}^{l}$. Using Proposition 5.14 to transfer the question to $\operatorname{Alg}_{\mathcal{O}^{\text {s }}}(\mathrm{Sym})$, all of the discussion above follows through by replacing uses of Theorems 1.2 and 1.3 by their Sym analogues, Propositions 5.44 and 5.43. The only caveat is that when running the second filtration argument in the proof (specifically, when analyzing (18)), one 
instead uses the BSym analogues mentioned in Remark 5.45.

\subsection{Proofs of Theorems 1.4, 1.5, 1.6 and 1.8}

We now derive Theorems 1.4, 1.5, 1.6, 1.8 from our main result, Theorem 1.1. Some of the proofs will make use of the following model structure on $\operatorname{Mod}_{\mathcal{O}}^{r}$.

Theorem 5.46. Let $\mathcal{O}$ be an operad in $\mathrm{Sp}^{\Sigma}$. There exists a cofibrantly generated model structure on $\operatorname{Mod}_{\mathcal{O}}^{r}$, which we call the monomorphism stable model structure, such that cofibrations and weak equivalences are underlying in the monomorphism stable model structure on Sym. Further, this is a left proper cellular simplicial model category.

Proof. This is a generalization of Theorem 3.7 and the same proof applies with only minor changes, hence we list only those.

Again one starts by proving a level equivalence result by verifying the conditions in [8, Thm. 2.1.19]. Choosing $\kappa$ to be an infinite cardinal larger than the number of simplices in $\mathcal{O}$ (counted over operadic, spectral and simplicial gradings), we define the set $I$ (resp., $J$ ) of generating cofibrations (resp., trivial cofibrations) to be a set of representatives of monomorphisms (resp., monomorphisms that are weak equivalences) between right modules with less than $\kappa$ simplices. Parts 1, 2, 3, 4 of $[\mathbf{8}$, Thm. 2.1.19] are again immediate, and part 5 follows by noting that $I$ contains the maps $\left(S \otimes\left(\Sigma_{m} \times \Sigma_{r} \cdot\left(\partial \Delta_{+}^{k} \rightarrow \Delta_{+}^{k}\right)\right)\right) \circ \mathcal{O}$. Part 6 reduces to showing a suitable " $\kappa$ analogue" of [9, Lemma 5.1.7], and again the proof in [9] generalizes by noting that all relative homotopy groups have less than $\kappa$ elements and by building the $F C$ subspectra as sub-right modules rather than just subspectra. Left properness, cellularity and the simplicial model structure axioms are again straightforward.

To produce the desired stable version one again applies [7, Thm. 4.1.1], now localizing with respect to the set $\mathcal{S}_{\mathcal{O}}=\bigcup_{r \geqslant 0}\left(\Sigma_{r} \cdot \mathcal{S}_{*}\right) \circ \mathcal{O}$. That the resulting weak equivalences are as described follows by arguing exactly as in the last paragraph of the proof of Theorem 3.6, using an identity adjunction to compare with the $\mathcal{O}$-projective model structure on $\operatorname{Mod}_{\mathcal{O}}^{r}$ over the $S$ stable model structure in Sym (this latter model structure is easily seen to exist by [15, Lemma 2.3]).

Proof of Theorem 1.4. To show the model structures exist it suffices (cf. [15, Lemma $2.3]$ ) to check that for $J$ a set of generating trivial cofibrations, any transfinite composition of pushouts of maps in $\mathcal{O} \circ J$ is a weak equivalence. Noting that the proof of Theorem 1.1 uses such a decomposition of $f_{2}$ and setting $f_{1}=* \rightarrow \mathcal{O}$ it is always the case that $f_{1} \circ_{\mathcal{O}} A$ is a monomorphism, so that repeating the first half of that proof one reduces to verifying that $*=*_{A} \rightarrow \mathcal{O}_{A}$ is a monomorphism, which is obviously the case even if $A$ is not cofibrant.

To verify the Quillen equivalence statement it suffices to show that the adjunction unit maps $A \rightarrow \overline{\mathcal{O}} \circ_{\mathcal{O}} A$, or $(\mathcal{O} \rightarrow \overline{\mathcal{O}}) \circ_{\mathcal{O}} A$, are weak equivalences whenever $A$ is cofibrant. Applying Theorem 1.1 with $f_{2}=\mathcal{O}(0) \rightarrow A$ and noting that $f \circ_{\mathcal{O}} \mathcal{O}(0)=$ $f \circ_{\mathcal{O}} \mathcal{O} \circ *=f \circ *=f(0)$ shows that the functor $(-) \circ_{\mathcal{O}} A$ preserves all weak equivalences that are also monomorphisms. It then follows from Theorem 5.46 combined with Ken Brown's lemma [7, Cor. 7.7.2] that $(-) \circ_{\mathcal{O}} A$ preserves all weak equivalences, finishing the proof. 
Proof of Theorem 1.5. Apply Theorem 1.1 to $f_{1}=(* \rightarrow \mathcal{O})$ and $f_{2}$ the intended cofibration between cofibrant objects.

Lemma 5.47. Consider positive $S$ cofibrations $f_{i}: A_{i} \rightarrow B_{i}, 1 \leqslant i \leqslant n$ in Sym all with positive $S$ cofibrant domains. Then their pushout product with respect to $\circ$,

$$
\square^{\circ}\left(f_{1}, f_{2}, \cdots, f_{n}\right)
$$

is a positive $S$ cofibration in Sym between positive $S$ cofibrant objects in Sym, which is a weak equivalence if any of the $f_{i}$ is.

Proof. The proof follows by induction on $n$.

The case $n=2$ is a essentially a particular case of Theorem 1.1 with $\mathcal{O}=\mathcal{I}$, except with an extra claim about positiveness. The extra claim follows by equation (4) which shows that $(X \circ Y)_{0}(0)=*$ if both $X_{0}(0)=*$ and $Y_{0}(0)=*$.

For the induction case, recalling that o preserves colimits in the first variable yields

$$
\left(f_{1}, f_{2}, \cdots, f_{n}\right)=\left(\square^{\circ}\left(f_{1}, f_{2}, \cdots, f_{n}-1\right)\right) \square^{\circ} f_{n}
$$

(note, however, that the similar equation with brackets on the right fails), and the result follows by combining the induction hypothesis with the $n=2$ case.

Proof of Theorem 1.6. Recall that the degeneracies of $B_{n}(M, \mathcal{O}, N)$ are formed using only the unit map $\eta: \mathcal{I} \rightarrow \mathcal{O}$. The result now follows from Lemma 5.47 since the maps whose cofibrancy must be verified are the maps

$$
\square^{\circ}(* \rightarrow M, \eta, \cdots, \eta, * \rightarrow N),
$$

where $\eta$ is allowed to appear any number of times.

Proof of Theorem 1.8. By the existence of the monomorphism (resp., $\mathcal{O}$-projective $S$ ) stable model structure on $\operatorname{Mod}_{\mathcal{O}}^{r}$ (cf. Theorem 5.46 (resp., its proof)) together with the fact that colimits (resp., limits) are underlying, homotopy cofiber (resp., fiber) sequences in $\operatorname{Mod}_{\mathcal{O}}^{r}$ match the underlying homotopy cofiber (resp., fiber) sequences in Sym. Therefore, homotopy fiber and cofiber sequences in $\operatorname{Mod}_{\mathcal{O}}^{r}$ coincide since Sym is stable. Noting that the argument in the proof of Theorem 1.4 shows $(-) \circ_{\mathcal{O}} A$ is already a left derived functor, and hence preserves homotopy cofiber sequences, finishes the proof.

\section{References}

[1] A.D. Elmendorf and M.A. Mandell. Rings, modules, and algebras in infinite loop space theory. Adv. Math., 205(1):163-228, 2006.

[2] P.G. Goerss and J.F. Jardine. Simplicial homotopy theory, volume of Mod. Birkhäuser Class. Birkhäuser Verlag, Basel, 2009. Reprint of the 1999 edition.

[3] J.E. Harper. Homotopy theory of modules over operads in symmetric spectra. Algebr. Geom. Topol., 9(3):1637-1680, 2009.

[4] J.E. Harper. Corrigendum to "Homotopy theory of modules over operads in symmetric spectra". Algebr. Geom. Topol., 15(2):1229-1237, 2015.

[5] J.E. Harper and K. Hess. Homotopy completion and topological Quillen homology of structured ring spectra. Geom. Topol., 17(3):1325-1416, 2013. 
[6] M. Hausmann. G-symmetric spectra, semistability and the multiplicative norm. Available at: http://http://arxiv.org/abs/1411.2290, 2014.

[7] P.S. Hirschhorn. Model categories and their localizations, volume 99 of Math. Surveys Monogr. Amer. Math. Soc., Providence, RI, 2003.

[8] M. Hovey. Model categories, volume 63 of Math. Surveys Monogr. Amer. Math. Soc., Providence, RI, 1999.

[9] M. Hovey, B. Shipley, and J. Smith. Symmetric spectra. J. Amer. Math. Soc., 13(1):149-208, 2000.

[10] S. Mac Lane. Categories for the working mathematician, volume 5 of Grad. Texts in Math.. Springer-Verlag, New York, second edition, 1998.

[11] M.A. Mandell, J.P. May, S. Schwede, and B. Shipley. Model categories of diagram spectra. Proc. Lond. Math. Soc. (3), 82(2):441-512, 2001.

[12] E. Riehl. Categorical homotopy theory, volume 24 of New Math. Monogr. Cambridge Univ. Press, Cambridge, 2014.

[13] E. Riehl and D. Verity. The theory and practice of Reedy categories. Theory Appl. Categ., 29:256-301, 2014.

[14] S. Schwede. An untitled book project about symmetric spectra. Available at: www.math.uni-bonn.de/people/schwede/SymSpec.pdf, 1997.

[15] S. Schwede and B.E. Shipley. Algebras and modules in monoidal model categories. Proc. Lond. Math. Soc. (3), 80(2):491-511, 2000.

[16] B. Shipley. A convenient model category for commutative ring spectra. In Homotopy theory: relations with algebraic geometry, group cohomology, and algebraic K-theory, volume 346 of Contemp. Math., pages 473-483. Amer. Math. Soc., Providence, RI, 2004.

[17] D. White. Model structures on commutative monoids in general model categories. Available at: http://arxiv.org/abs/1403.6759, 2014.

[18] D. White and D. Yau. Bousfield localization and algebras over colored operads. Available at: http://arxiv.org/abs/1503.06720, 2015.

Luís Alexandre Pereira lp2h@virginia.edu

Mathematics Department, University of Virginia, Charlottesville, VA, 22904, USA 